\title{
The Lie algebraic significance of symmetric informationally complete measurements
}

\author{
D. M. Appleby, Steven T. Flammia, ${ }^{\text {a) }}$ and Christopher A. Fuchs \\ Perimeter Institute for Theoretical Physics, Waterloo, Ontario N2L 2Y5, Canada
}

(Received 15 February 2010; accepted 26 January 2011; published online 25 February 2011)

\begin{abstract}
Examples of symmetric informationally complete positive operator-valued measures (SIC-POVMs) have been constructed in every dimension $\leq 67$. However, it remains an open question whether they exist in all finite dimensions. A SIC-POVM is usually thought of as a highly symmetric structure in quantum state space. However, its elements can equally well be regarded as a basis for the Lie algebra $\operatorname{gl}(d, \mathbb{C})$. In this paper we examine the resulting structure constants, which are calculated from the traces of the triple products of the SIC-POVM elements and which, it turns out, characterize the SIC-POVM up to unitary equivalence. We show that the structure constants have numerous remarkable properties. In particular we show that the existence of a SIC-POVM in dimension $d$ is equivalent to the existence of a certain structure in the adjoint representation of $\operatorname{gl}(d, \mathbb{C})$. We hope that transforming the problem in this way, from a question about quantum state space to a question about Lie algebras, may help to make the existence problem tractable. (c) 2011 American Institute of Physics. [doi:10.1063/1.3555805]
\end{abstract}

\section{INTRODUCTION}

Symmetric informationally complete positive operator-valued measures (SIC-POVMs) present us with what is, simultaneously, one of the most interesting and one of the most difficult and tantalizing problems in quantum information. ${ }^{1-46}$ SIC-POVMs are important, practically, with applications to quantum tomography and cryptography, ${ }^{4,8,12,15,20,29}$ and to classical signal processing. ${ }^{24,36}$ However, without in any way wishing to impugn the significance of the applications which have so far been proposed, it appears to us that the interest of SIC-POVMs stems less from these particular proposed uses than from rather broader, more general considerations: the sense one gets that SICs are telling us something deep and hitherto unsuspected about the structure of quantum state space. In spite of its being the central object about which the rest of quantum mechanics rotates, and notwithstanding the efforts of numerous investigators, ${ }^{47}$ the geometry of the quantum state space continues to be surprisingly ill-understood. The hope which inspires our efforts is that a solution to the SIC problem will prove to be the key, not just to the SIC-POVMs narrowly conceived but to the geometry of state space in general. Such things are, by nature, unpredictable. However, it is not unreasonable to speculate that a better theoretical understanding of the geometry of quantum state space might have important practical consequences: not only the applications listed above but perhaps other applications which have yet to be conceived. On a more foundational level one may hope that it will lead to a much improved understanding of the conceptual message of quantum mechanics. ${ }^{7,43,45,48}$

Having said why we describe the problem as interesting, let us now explain why we describe it as tantalizing. The trouble is that, although there is an abundance of reasons for suspecting that SIC-POVMs exist in every finite dimension (exact and high-precision numerical examples $1,2,5,11,16,19,28,39,46$ having now been constructed in every dimension up to 67), and in

\footnotetext{
a) Author to whom correspondence should be addressed. Electronic mail: sflammia @ perimeterinstitute.ca. Permanent address: California Institute of Technology, Institute for Quantum Information, Pasadena, California 91125, USA.
} 
spite of the intense efforts of many people ${ }^{1-46}$ extending over a period of more than ten years, a general existence proof continues to elude us. In their seminal paper on the subject, published in 2004, Renes et al. ${ }^{5}$ say "A rigorous proof of existence of SIC-POVMs in all finite dimensions seems tantalizingly close, yet remains somehow distant." They could have said the same if they were writing today.

The purpose of this paper is to try to take our understanding of SIC mathematics (as it might be called) a little further forward. The research we report began with a chance numerical discovery made while we were working on a different problem. Pursuing that initial numerical hint, we uncovered a rich and interesting set of connections between SIC-POVMs in dimension $d$ and the Lie algebra $\operatorname{gl}(d, \mathbb{C})$. The existence of these connections came as a surprise to us. However, in retrospect it is, perhaps, not so surprising. Interest in SIC-POVMs has, to date, focused on the fact that an arbitrary density matrix can be expanded in terms of a SIC-POVM. However, a SIC-POVM in dimension $d$ does in fact provide a basis, not just for the space of density matrices but for the space of all $d \times d$ complex matrices-i.e., the Lie algebra $\mathrm{gl}(d, \mathbb{C})$. Boykin et al. ${ }^{49}$ have recently shown that there is a connection between the existence problem for maximal sets of mutually unbiased bases (MUBs) and the theory of Lie algebras. Since SIC-POVMs share the property of being highly symmetrical structures in quantum state space with MUBs, it might have been anticipated that there are also some interesting connections between SIC-POVMs and Lie algebras.

Our main result (proved in Secs. IV-VI) is that the proposition, that a SIC-POVM exists in dimension $d$, is equivalent to a proposition about the adjoint representation of $\operatorname{gl}(d, \mathbb{C})$, in particular to the existence of a highly symmetrical basis for this Lie algebra. Our hope is that transforming the problem in this way, from a question about quantum state space to a question about Lie algebras, may help to make the SIC-existence problem tractable. But even if this hope fails to materialize we feel that this result, along with the many other results we obtain, provides some additional insight into these structures.

In order to state our main results more clearly, we first review the definition of a SIC-POVM and develop some notation for the elements of the Lie algebra which will be our primary objects of study.

\section{BACKGROUND, NOTATION, AND SUMMARY OF MAIN RESULTS}

\section{A. Background on SIC-POVMs}

In $d$ dimensional Hilbert space $\mathcal{H}_{d}$, a SIC-POVM is a set of $d^{2}$ operators $E_{1}, \ldots, E_{d^{2}}$ of the form

$$
E_{r}=\frac{1}{d} \Pi_{r}
$$

where the $\Pi_{r}$ are rank-1 projectors with the property

$$
\operatorname{Tr}\left(\Pi_{r} \Pi_{s}\right)=\left\{\begin{array}{ll}
1 & r=s \\
\frac{1}{d+1} & r \neq s
\end{array} .\right.
$$

We will refer to the $\Pi_{r}$ as SIC-projectors, and we say that $\left\{\Pi_{r}: r=1, \ldots, d^{2}\right\}$ is a SIC set.

It follows from this definition that the $E_{r}$ satisfy

$$
\sum_{r} E_{r}=I
$$

[so they constitute a positive operator-valued measure (POVM)] and that they are linearly independent (so the POVM is informationally complete).

It is an open question whether SIC-POVMs exist for all values of $d$. However, examples have been constructed analytically in dimensions $2-15$ inclusive, ${ }^{1,2,11,16,19,28,39,46}$ and in dimensions 19, 24, 35, and 48. ${ }^{16,46}$ Moreover, high precision numerical solutions have been constructed in dimensions 2-67 inclusive. ${ }^{5,46}$ This lends some plausibility to the speculation that they exist in all dimensions. For a comprehensive account of the current state of knowledge in this regard and many new results, see the recent study by Scott and Grassl. ${ }^{46}$ 
All known SIC-POVMs have a group covariance property. In other words, there exists

1. a group $G$ having $d^{2}$ elements,

2. a projective unitary representation of $G$ on $\mathcal{H}_{d}$ : i.e., a map $g \rightarrow U_{g}$ from $G$ to the set of unitaries such that $U_{g_{1}} U_{g_{2}} \sim U_{g_{1} g_{2}}$ for all $g_{1}, g_{2}$ (where the notation " " means "equals up to a phase"), and

3. a normalized vector $|\psi\rangle$ (the fiducial vector),

such that the SIC-projectors are given by

$$
\Pi_{g}=U_{g}|\psi\rangle\langle\psi| U_{g}^{\dagger}
$$

(where we label the projector by the group element $g$, rather than the integer $r$ as above).

Most known SIC-POVMs are covariant under the action of the Weyl-Heisenberg group (though not all-see Renes et al. $^{5}$ and, for an explicit example of a non-Weyl-Heisenberg SIC-POVM, see Grass $\left.{ }^{19}\right)$. Here the group is $\mathbb{Z}_{d} \times \mathbb{Z}_{d}$, and the projective representation is $\mathbf{p} \rightarrow D_{\mathbf{p}}$, where $\mathbf{p}=\left(p_{1}, p_{2}\right) \in \mathbb{Z}_{d} \times \mathbb{Z}_{d}$ and $D_{\mathbf{p}}$ is the corresponding Weyl-Heisenberg displacement operator

$$
D_{\mathbf{p}}=\sum_{r=0}^{d-1} \tau^{\left(2 r+p_{1}\right) p_{2}}\left|r+p_{1}\right\rangle\langle r| .
$$

In this expression $\tau=e^{\frac{i \pi(d+1)}{d}}$, the vectors $|0\rangle, \ldots|d-1\rangle$ are an orthonormal basis, and the addition in $\left|r+p_{1}\right\rangle$ is modulo $d$. For more details, see, for example, Ref. 16 .

One should not attach too much weight to the fact that all known SIC-POVMs have a group covariance property as this may only reflect the fact that group covariant SIC-POVMs are much easier to construct. So in this paper we will try to prove as much as we can without assuming such a property. One potential benefit of this attitude is that, by accumulating enough facts about SIC-POVMs in general, we may eventually get to the point where we can answer the question, whether all SIC-POVMs actually do have a group covariance property.

\section{B. Lie algebras and some notation}

The fact that the $d^{2}$ operators $\Pi_{r}$ are linearly independent means that they form a basis for the complex Lie algebra $\operatorname{gl}(d, \mathbb{C})$ (the set of all operators acting on $\mathcal{H}_{d}$ ). Since the $\Pi_{r}$ are Hermitian, then $i \Pi_{r}$ forms a basis also for the real Lie algebra $\mathrm{u}(d)$ (the set of all anti-Hermitian operators acting on $\left.\mathcal{H}_{d}\right)$. So for any operator $A \in \operatorname{gl}(d, \mathbb{C})$ there is a unique set of expansion coefficients $a_{r}$ such that

$$
A=\sum_{r} a_{r} \Pi_{r}
$$

To find the expansion coefficients we can use the fact that

$$
\sum_{s} \operatorname{Tr}\left(\Pi_{r} \Pi_{s}\right)\left(\frac{d+1}{d} \delta_{s t}-\frac{1}{d^{2}}\right)=\delta_{r t},
$$

from which it follows:

$$
a_{r}=\frac{d+1}{d} \operatorname{Tr}\left(\Pi_{r} A\right)-\frac{1}{d} \operatorname{Tr}(A) .
$$

Specializing to the case $A=\Pi_{r} \Pi_{s}$, we find

$$
\Pi_{r} \Pi_{s}=\frac{d+1}{d}\left(\sum_{t} T_{r s t} \Pi_{t}\right)-\frac{d \delta_{r s}+1}{d+1} I,
$$

where

$$
T_{r s t}=\operatorname{Tr}\left(\Pi_{r} \Pi_{s} \Pi_{t}\right)
$$


To a large extent this paper consists of an exploration of the properties of these important quantities, which we will refer to as the triple products. They are intimately related to the geometric phase, in which context they are usually referred to as 3-vertex Bargmann invariants (see Mukunda et al. ${ }^{50}$ and references cited therein). We have, as an immediate consequence of the definition,

$$
T_{r s t}=T_{t r s}=T_{s t r}=T_{r t s}^{*}=T_{t s r}^{*}=T_{s r t}^{*} .
$$

It is convenient to define

$$
\begin{aligned}
& J_{r s t}=\frac{d+1}{d}\left(T_{r s t}-T_{r s t}^{*}\right), \\
& R_{r s t}=\frac{d+1}{d}\left(T_{r s t}+T_{r s t}^{*}\right) .
\end{aligned}
$$

So $J_{r s t}$ is imaginary and completely antisymmetric; $R_{r s t}$ is real and completely symmetric. Both these quantities play a significant role in the theory. It follows from Eq. (9) that

$$
\left[\Pi_{r}, \Pi_{s}\right]=\sum_{t} J_{r s t} \Pi_{t} .
$$

So the $J_{r s t}$ are structure constants for the Lie algebra $\mathrm{gl}(d, \mathbb{C})$. As an immediate consequence of this they satisfy the Jacobi identity

$$
\sum_{b}\left(J_{r s b} J_{t b a}+J_{s t b} J_{r b a}+J_{t r b} J_{s b a}\right)=0
$$

for all $r, s, t, a$. The Jacobi identity holds for any representation of the structure constants. In Secs. III-IX we will derive many other identities which are specific to this particular representation.

Turning to the quantities $R_{r s t}$, it follows from Eq. (9) that they feature in the expression for the anticommutator

$$
\left\{\Pi_{r}, \Pi_{s}\right\}=\sum_{t} R_{r s t} \Pi_{t}-\frac{2\left(d \delta_{r s}+1\right)}{d+1} I .
$$

They also play an important role in the description of quantum state space. Let $\rho$ be any density matrix and let $p_{r}=\frac{1}{d} \operatorname{Tr}\left(\Pi_{r} \rho\right)$ be the probability of obtaining outcome $r$ in the measurement described by the POVM with elements $\frac{1}{d} \Pi_{r}$. Then it follows from Eq. (8) that $\rho$ can be reconstructed from the probabilities by

$$
\rho=\sum_{r}\left((d+1) p_{r}-\frac{1}{d}\right) \Pi_{r} .
$$

Suppose, now, that the $p_{r}$ are any set of $d^{2}$ real numbers. So we do not assume that the $p_{r}$ are even probabilities, let alone the probabilities coming from a density matrix according to the prescription $p_{r}=\frac{1}{d} \operatorname{Tr}\left(\Pi_{r} \rho\right)$. Then it is shown in Ref. 34 that the $p_{r}$ are in fact the probabilities coming from a pure state if and only if they satisfy the following two conditions:

$$
\begin{gathered}
\sum_{r} p_{r}^{2}=\frac{2}{d(d+1)} \\
\sum_{r, s, t} R_{r s t} p_{r} p_{s} p_{t}=\frac{2(d+7)}{d(d+1)^{2}} .
\end{gathered}
$$

Let us look at the quantities $J_{r s t}$ and $R_{r s t}$ in a little more detail. For each $r$ choose a unit vector $\left|\psi_{r}\right\rangle$ such that $\Pi_{r}=\left|\psi_{r}\right\rangle\left\langle\psi_{r}\right|$. Then the Gram matrix for these vectors is of the form

$$
G_{r s}=\left\langle\psi_{r} \mid \psi_{s}\right\rangle=K_{r s} e^{i \theta_{r s}},
$$


where the matrix $\theta_{r s}$ is antisymmetric and

$$
K_{r s}=\sqrt{\frac{d \delta_{r s}+1}{d+1}} .
$$

Note that the SIC-POVM does not determine the angles $\theta_{r s}$ uniquely since making the replacements $\left|\psi_{r}\right\rangle \rightarrow e^{i \phi_{r}}\left|\psi_{r}\right\rangle$ leaves the SIC-POVM unaltered, but changes the angles $\theta_{r s}$ according to the prescription $\theta_{r s} \rightarrow \theta_{r s}-\phi_{r}+\phi_{s}$. This freedom to rephase the vectors $\left|\psi_{r}\right\rangle$ is not usually important. However, it sometimes has interesting consequences (see Sec. X). It can be thought of as a kind of gauge freedom.

The Gram matrix satisfies an important identity. Every SIC-POVM has the 2-design property, ${ }^{5,17}$

$$
\sum_{r} \Pi_{r} \otimes \Pi_{r}=\frac{2 d}{d+1} P_{\mathrm{sym}}
$$

where $P_{\text {sym }}$ is the projector onto the symmetric subspace of $\mathcal{H}_{d} \otimes \mathcal{H}_{d}$. Expressed in terms of the Gram matrix this becomes

$$
\sum_{r} G_{s_{1} r} G_{s_{2} r} G_{r t_{1}} G_{r t_{2}}=\frac{d}{d+1}\left(G_{s_{1} t_{1}} G_{s_{2} t_{2}}+G_{s_{1} t_{2}} G_{s_{2} t_{1}}\right) .
$$

Turning to the triple products we have

$$
T_{r s t}=G_{r s} G_{s t} G_{t r}=K_{r s} K_{s t} K_{t r} e^{i \theta_{r s t}}
$$

where

$$
\theta_{r s t}=\theta_{r s}+\theta_{s t}+\theta_{t r}
$$

Note that the tensor $\theta_{r s t}$ is completely antisymmetric. In particular $\theta_{r s t}=0$ if any of the two indices are the same. Also note that rephasing the vectors $\left|\psi_{r}\right\rangle$ leaves the tensors $T_{r s t}$ and $\theta_{r s t}$ unchanged. They are in that sense gauge invariant.

Finally, we have the following expressions for $J_{r s t}$ and $R_{r s t}$ :

$$
\begin{gathered}
J_{r s t}=\frac{2 i}{d \sqrt{d+1}} \sin \theta_{r s t}, \\
R_{r s t}=\frac{2(d+1)}{d} K_{r s} K_{s t} K_{t r} \cos \theta_{r s t .}
\end{gathered}
$$

Like the triple products, $J_{r s t}$ and $R_{r s t}$ are gauge invariant.

For later reference let us note that the matrix $J_{r}$, with matrix elements

$$
\left(J_{r}\right)_{s t}=J_{r s t},
$$

is the adjoint representative of $\Pi_{r}$ in the SIC-projector basis,

$$
\operatorname{ad}_{\Pi_{r}} \Pi_{s}=\left[\Pi_{r}, \Pi_{s}\right]=\sum_{t} J_{r s t} \Pi_{t} .
$$

It can be seen that all the interesting features of the tensor $G_{r s}$ (respectively, the tensors $T_{r s t}$, $J_{r s t}$, and $R_{r s t}$ ) are contained in the order- 2 angle tensor $\theta_{r s}$ (respectively, the order-3 angle tensor $\left.\theta_{r s t}\right)$. It is also easy to see that, for any unitary $U$, the transformation,

$$
\Pi_{r} \rightarrow U \Pi_{r} U^{\dagger},
$$

leaves the angle tensors invariant. This suggests that we shift our focus from individual SIC-POVMs to families of unitarily equivalent SIC-POVMs_-SIC-families, as we will call them for short. 


\section{Overview and main results}

We begin our investigation in Sec. III by giving necessary and sufficient conditions for an arbitrary tensor $\theta_{r s}$ (respectively, $\theta_{r s t}$ ) to be the rank-2 (respectively, rank-3) angle tensor corresponding to a SIC-family. We also show that either angle tensor uniquely determines the corresponding SICfamily. Finally we describe a method for reconstructing the SIC-family, starting from a knowledge of either of the two angle tensors.

In Secs. IV-VI we prove the central result of this paper: namely, that the existence of a SICPOVM in dimension $d$ is equivalent to the existence of a certain very special set of matrices in the adjoint representation of $\operatorname{gl}(d, \mathbb{C})$. In Sec. IV we show that, for any SIC-POVM, the adjoint matrices $J_{r}$ have the spectral decomposition

$$
J_{r}=Q_{r}-Q_{r}^{\mathrm{T}},
$$

where $Q_{r}$ is a rank $d-1$ projector which has the remarkable property of being orthogonal to its own transpose,

$$
Q_{r} Q_{r}^{\mathrm{T}}=0 .
$$

We refer to this feature of the adjoint matrices as the $Q-Q^{\mathrm{T}}$ property. In Sec. IV we also show that from a knowledge of the $J$ matrices it is possible to reconstruct the corresponding SIC-family. In Sec. V we characterize the general class of projectors which have the property of being orthogonal to their own transpose. Then, in Sec. VI, we prove a converse of the result established in Sec. IV. The $Q-Q^{\mathrm{T}}$ property is not completely equivalent to the property of being a SIC set. However, it turns out that it is, in a certain sense, very nearly equivalent. To be more specific, let $L_{r}$ be any set of $d^{2}$ Hermitian operators which constitute a basis for $\operatorname{gl}(d, \mathbb{C})$ and let $C_{r}$ be the adjoint representative of $L_{r}$ in this basis. Then the necessary and sufficient condition for the $C_{r}$ to have the spectral decomposition,

$$
C_{r}=Q_{r}-Q_{r}^{\mathrm{T}},
$$

where $Q_{r}$ is a rank $d-1$ projector such that $Q_{r} Q_{r}^{\mathrm{T}}=0$ is that there exists a SIC set $\Pi_{r}$ such that $L_{r}=\epsilon_{r}\left(\Pi_{r}+\alpha I\right)$ for some fixed number $\alpha \in \mathbb{R}$ and signs $\epsilon_{r}= \pm 1$. In particular, the existence of a Hermitian basis for $\operatorname{gl}(d, \mathbb{C})$ having the $Q-Q^{\mathrm{T}}$ property is both necessary and sufficient for the existence of a SIC-POVM in dimension $d$.

In Sec. VII we digress briefly and consider $\operatorname{sl}(d, \mathbb{C})$ (the Lie algebra consisting of all tracezero $d \times d$ complex matrices). As we have explained, this paper is motivated by the hope that a Lie algebraic perspective will cast light on the SIC-existence problem, rather than by an interest in Lie algebras as such. We focus on $\operatorname{gl}(d, \mathbb{C})$ because that is the case where the connection with SIC-POVMs seems most straightforward. However a SIC-POVM also gives rise to an interesting geometrical structure in $\operatorname{sl}(d, \mathbb{C})$, as we show in Sec. VII.

In Sec. VIII we derive a number of additional identities satisfied by the $J$ and $Q$ matrices.

The complex projectors $Q_{r}, Q_{r}^{\mathrm{T}}$, and the real projector $Q_{r}+Q_{r}^{\mathrm{T}}$ define three families of subspaces. It turns out that there are some interesting geometrical relationships between these subspaces, which we study in Sec. IX.

Finally, in Sec. X we show that, with the appropriate choice of gauge, the Gram matrix corresponding to a Weyl-Heisenberg covariant SIC-family has a feature analogous to the $Q-Q^{\mathrm{T}}$ property, which we call the $P-P^{\mathrm{T}}$ property. It is an open question whether this result generalizes to other SICfamilies, not covariant with respect to the Weyl-Heisenberg group.

\section{THE ANGLE TENSORS}

The purpose of this section is to establish the necessary and sufficient conditions for an arbitrary tensor $\theta_{r s}$ (respectively, $\theta_{r s t}$ ) to be the order-2 (respectively, order-3) angle tensor for a SIC-family. We will also show that either one of the angle tensors is enough to uniquely determine the SICfamily. Moreover, we will describe explicit procedures for reconstructing the family, starting from a knowledge of one of the angle tensors. 
We begin by considering the general class of POVMs (not just SIC-POVMs) which consist of $d^{2}$ rank-1 elements. A POVM of this type is thus defined by a set of $d^{2}$ vectors $\left|\xi_{1}\right\rangle, \ldots,\left|\xi_{d^{2}}\right\rangle$ with the property

$$
\sum_{r}\left|\xi_{r}\right\rangle\left\langle\xi_{r}\right|=I
$$

Note that $\sum_{r} \|\left|\xi_{r}\right\rangle \|^{2}=d$, so the vectors $\left|\xi_{r}\right\rangle$ cannot all be normalized. In the particular case of a SIC-POVM all the vectors have the same norm $\|\left|\xi_{r}\right\rangle \|=\frac{1}{\sqrt{d}}$. However in the general case they may have different norms.

Given a set of such vectors consider the Gram matrix

$$
P_{r s}=\left\langle\xi_{r} \mid \xi_{s}\right\rangle \text {. }
$$

Clearly the Gram matrix cannot determine the POVM uniquely since if $U$ is any unitary operator, then the vectors $U\left|\xi_{r}\right\rangle$ will define another POVM having the same Gram matrix. However, the theorem we now prove shows that this is the only freedom. In other words, the Gram matrix fixes the POVM up to unitary equivalence. The theorem also provides us with a criterion for deciding whether an arbitrary $d^{2} \times d^{2}$ matrix $P$ is the Gram matrix corresponding to a POVM of the specified type. As a corollary this will give us a criterion for deciding whether an arbitrary tensor $\theta_{r s}$ is specifically the order-2 angle tensor for a SIC-family.

Theorem 1: Let $P$ be any $d^{2} \times d^{2}$ Hermitian matrix. Then the following conditions are equivalent.

(1) $P$ is a rank-d projector.

(2) P satisfies the trace identities

$$
\operatorname{Tr}(P)=\operatorname{Tr}\left(P^{2}\right)=\operatorname{Tr}\left(P^{3}\right)=\operatorname{Tr}\left(P^{4}\right)=d .
$$

(3) $P$ is the Gram matrix for a set of $d^{2}$ vectors $\left|\xi_{r}\right\rangle$ (not all normalized) such that $\left|\xi_{r}\right\rangle\left\langle\xi_{r}\right|$ is a POVM, satisfying in particular Eqs. (34) and (35).

Suppose P satisfies these conditions. To construct a POVM corresponding to P let the d column vectors,

$$
\hat{\xi}=\left(\begin{array}{c}
\xi_{11} \\
\xi_{12} \\
\vdots \\
\xi_{1 d^{2}}
\end{array}\right),\left(\begin{array}{c}
\xi_{21} \\
\xi_{22} \\
\vdots \\
\xi_{2 d^{2}}
\end{array}\right), \ldots,\left(\begin{array}{c}
\xi_{d 1} \\
\xi_{d 2} \\
\vdots \\
\xi_{d d^{2}}
\end{array}\right)
$$

be any orthonormal basis for the subspace onto which P projects. Define

$$
\left|\xi_{r}\right\rangle=\sum_{a=1}^{d} \xi_{a r}^{*}|a\rangle,
$$

where the vectors $|a\rangle$ are any orthonormal basis for $\mathcal{H}_{d}$. Then $P$ is the Gram matrix for the vectors $\left|\xi_{1}\right\rangle, \ldots,\left|\xi_{d^{2}}\right\rangle$. Moreover, the necessary and sufficient condition for any other set of vectors $\left|\eta_{1}\right\rangle, \ldots,\left|\eta_{d^{2}}\right\rangle$ to have Gram matrix $P$ is that there exists a unitary operator $U$ such that

$$
\left|\eta_{r}\right\rangle=U\left|\xi_{r}\right\rangle
$$

for all $r$.

Proof: We begin by showing that (3) $\Rightarrow(1)$. Suppose $\left|\xi_{1}\right\rangle, \ldots\left|\xi_{d^{2}}\right\rangle$ is any set of $d^{2}$ vectors such that $\left|\xi_{r}\right\rangle\left\langle\xi_{r}\right|$ is a POVM so that the completeness relation Eq. (34) holds, and let $P_{r s}=\left\langle\xi_{r} \mid \xi_{s}\right\rangle$ be the 
Gram matrix. Then $P$ is a Hermitian. Moreover, $P^{2}=P$ since

$$
\sum_{t} P_{r t} P_{t s}=\left\langle\xi_{r}\left|\left(\sum_{t}\left|\xi_{t}\right\rangle\left\langle\xi_{s}\right|\right)\right| \xi_{r}\right\rangle=\left\langle\xi_{r} \mid \xi_{s}\right\rangle=P_{r s}
$$

By taking the trace of the completeness relation, we conclude that $P$ is a rank- $d$ projector.

We next show that $(1) \Rightarrow(3)$. Let $P$ be a rank- $d$ projector, and let the $d$ column vectors $\hat{\xi}$ from Eq. (37) be an orthonormal basis for the subspace onto which it projects. So for all $a, b$, and for all $r, s$ we have

$$
\sum_{r} \xi_{a r}^{*} \xi_{b r}=\delta_{a b} \quad \text { and } \quad \sum_{a} \xi_{a r} \xi_{a s}^{*}=P_{r s}
$$

Now let $\left|\xi_{1}\right\rangle, \ldots\left|\xi_{d^{2}}\right\rangle$ be the vectors defined by Eq. (38), and the conditions Eq. (34) and Eq. (35) follow easily, showing that the $\left|\xi_{r}\right\rangle$ are a POVM with the requisite Gram matrix.

We next turn to condition (2). The fact that $(1) \Rightarrow(2)$ is immediate. To prove the reverse implication observe that condition (2) implies

$$
\operatorname{Tr}\left(P^{4}\right)-2 \operatorname{Tr}\left(P^{3}\right)+\operatorname{Tr}\left(P^{2}\right)=0 .
$$

Since $P$ is a Hermitian, this is equivalent to $\operatorname{Tr}\left(X^{*} X\right)=0$, where $X=P(I-P)$; hence, $X=0$ and $P$ is a rank- $d$ projector.

It remains to show that the POVM corresponding to a given rank- $d$ projector is unique up to unitary equivalence. To prove this let $P$ be a rank- $d$ projector, let $\left|\xi_{r}\right\rangle$ be the vectors defined by Eq. (38), and let $\left|\eta_{1}\right\rangle, \ldots,\left|\eta_{d^{2}}\right\rangle$ be any other set of vectors such that $\left\langle\eta_{r} \mid \eta_{s}\right\rangle=P_{r s}$ for all $r, s$; also let $\eta_{a r}=\left\langle\eta_{r} \mid a\right\rangle$. Then

$$
\sum_{r} \eta_{a r}^{*} \eta_{b r}=\left\langle a\left|\left(\sum_{r}\left|\eta_{r}\right\rangle\left\langle\eta_{r}\right|\right)\right| b\right\rangle=\delta_{a b}
$$

(because $\left|\eta_{r}\right\rangle\left\langle\eta_{r}\right|$ is a POVM) and

$$
\sum_{a=1}^{d} \eta_{a r} \eta_{a s}^{*}=P_{r s}
$$

(because the $\left|\eta_{r}\right\rangle$ have Gram matrix $P$ ). So the $d$ column vectors $\hat{\eta}$, defined in analogy with Eq. (37), are an orthonormal basis for the subspace onto which $P$ projects. But the column vectors $\hat{\xi}$ are also an orthonormal basis for this subspace. So there must exist a $d \times d$ unitary matrix $U_{a b}$ such that

$$
\eta_{a r}=\sum_{b=1}^{d} U_{a b} \xi_{b r}
$$

for all $a, r$. Defining

$$
U=\sum_{a, b=1}^{d} U_{a b}^{*}|a\rangle\langle b|,
$$

we have $\left|\eta_{r}\right\rangle=U\left|\xi_{r}\right\rangle$ for all $r$.

In the case of a SIC-POVM we have

$$
\left|\xi_{r}\right\rangle=\frac{1}{\sqrt{d}}\left|\psi_{r}\right\rangle
$$

where the vectors $\left|\psi_{r}\right\rangle$ are normalized, and

$$
P_{r s}=\frac{1}{d} G_{r s}=\frac{1}{d} K_{r s} e^{i \theta_{r s}},
$$


where $G$ is the Gram matrix of the vectors $\left|\psi_{r}\right\rangle$ and $\theta_{r s}$ is the order-2 angle tensor. In the sequel, we will distinguish these matrices by referring to $G$ as the Gram matrix and $P$ as the Gram projector. We have

Corollary 2: Let $\theta_{\text {rs }}$ be a real antisymmetric tensor. Then the following statements are equivalent.

1. $\theta_{r s}$ is an order-2 angle tensor corresponding to a SIC-family.

2. $\theta_{\text {rs }}$ satisfies

$$
\sum_{t} K_{r t} K_{t s} e^{i\left(\theta_{r t}+\theta_{t s}\right)}=d K_{r s} e^{i \theta_{r s}}
$$

for all $r, s$.

3. $\theta_{r s}$ satisfies

$$
\begin{aligned}
\sum_{r, s, t} K_{r s} K_{s t} K_{t r} e^{i\left(\theta_{r s}+\theta_{s t}+\theta_{t r}\right)} & =d^{4}, \\
\sum_{r, s, t, u} K_{r s} K_{s t} K_{t u} K_{u r} e^{i\left(\theta_{r s}+\theta_{s t}+\theta_{t u}+\theta_{u r}\right)} & =d^{5} .
\end{aligned}
$$

Let $\Pi_{r}$ and $\Pi_{r}^{\prime}$ be two different SIC-sets and let $\theta_{r s}$ and $\theta_{r s}^{\prime}$ be corresponding order-2 angle tensors. Then there exists a unitary $U$ such that

$$
\Pi_{r}^{\prime}=U \Pi_{r} U^{\dagger}
$$

for all $r$ if and only if

$$
\theta_{r s}^{\prime}=\theta_{r s}-\phi_{r}+\phi_{s},
$$

for some arbitrary set of phase angles $\phi_{r}$ (in other words two SIC-sets are unitarily equivalent if and only if their order- 2 angle tensors are gauge equivalent).

A SIC-family can be reconstructed from its order-2 angle tensor $\theta_{r s}$ by calculating an orthonormal basis for the subspace onto which the Gram projector,

$$
P_{r s}=\frac{1}{d} K_{r s} e^{i \theta_{r s}}
$$

projects, as described in Theorem 1.

Remark: The sense in which we are using the term "gauge equivalence" is explained in the paragraph immediately following Eq. (21).

Note that condition (2) imposes $d^{2}\left(d^{2}-1\right) / 2$ independent constraints (taking account of the antisymmetry of $\theta_{r s}$ ). Condition (3), by contrast, only imposes two independent constraints. It is to be observed, however, that the price we pay for the reduction in the number of equations is that Eqs. (50) and (50) are, respectively, cubic and quartic in the phases, whereas Eq. (49) is only quadratic.

Proof: Let $\theta_{r s}$ be an arbitrary antisymmetric tensor and define

$$
P_{r s}=\frac{1}{d} K_{r s} e^{i \theta_{r s}}
$$

The antisymmetry of $\theta_{r s}$ means that $P$ is automatically Hermitian. So it follows from Theorem 1 that a necessary and sufficient condition for $P_{r s}$ to be a rank- $d$ projector, and for $\theta_{r s}$ to be the order- 2 angle tensor of a SIC-family, is that

$$
\sum_{t} K_{r t} K_{t s} e^{i\left(\theta_{r t}+\theta_{t s}\right)}=d K_{r s} e^{i \theta_{r s}}
$$

for all $r, s$. 
To prove the equivalence of conditions (1) and (3) note that the conditions $\operatorname{Tr}(P)=\operatorname{Tr}\left(P^{2}\right)=d$ are an automatic consequence of $P$ having the specified form. So it follows from Theorem 1 that $\theta_{r s}$ is the order-2 angle tensor of a SIC-family if and only if Eqs. (50) and (51) are satisfied.

Now let $\Pi_{r}$ and $\Pi_{r}^{\prime}$ be two SIC-sets and let $\theta_{r s}$ and $\theta_{r s}^{\prime}$ be order-2 angle tensors corresponding to them. Then there exist normalized vectors $\left|\psi_{r}\right\rangle$ and $\left|\psi_{r}^{\prime}\right\rangle$ such that $\Pi_{r}=\left|\psi_{r}\right\rangle\left\langle\psi_{r}\right|$ and $\Pi_{r}^{\prime}=\left|\psi_{r}^{\prime}\right\rangle\left\langle\psi_{r}^{\prime}\right|$ for all $r$, and $\left\langle\psi_{r} \mid \psi_{s}\right\rangle=K_{r s} e^{i \theta_{r s}}$ and $\left\langle\psi_{r}^{\prime} \mid \psi_{s}^{\prime}\right\rangle=K_{r s} e^{i \theta_{r s}^{\prime}}$ for all $r, s$.

Suppose, first of all, that there exists a unitary $U$ such that $\Pi_{r}^{\prime}=U \Pi_{r} U^{\dagger}$. Then there exist phase angles $\phi_{r}$ such that $\left|\psi_{r}^{\prime}\right\rangle=e^{i \phi_{r}} U\left|\psi_{r}\right\rangle$ for all $r$, which is easily seen to imply that $\theta_{r s}^{\prime}=\theta_{r s}-\phi_{r}+\phi_{s}$ for all $r, s$. So $\theta_{r s}$ and $\theta_{r s}^{\prime}$ are gauge equivalent.

Conversely, suppose there exist phase angles $\phi_{r}$ such that $\theta_{r s}^{\prime}=\theta_{r s}-\phi_{r}+\phi_{s}$. Define $\left|\psi_{r}^{\prime \prime}\right\rangle=$ $e^{-i \phi_{r}}\left|\psi_{r}^{\prime}\right\rangle$. Then $\left\langle\psi_{r}^{\prime \prime} \mid \psi_{s}^{\prime \prime}\right\rangle=K_{r s} e^{i \theta_{r s}}=\left\langle\psi_{r} \mid \psi_{s}\right\rangle$ for all $r, s$. So it follows from Theorem 1 that there exists a unitary $U$ such that $\left|\psi_{r}^{\prime \prime}\right\rangle=U\left|\psi_{r}\right\rangle$ for all $r$. Consequently, $\Pi_{r}^{\prime}=\left|\psi_{r}^{\prime \prime}\right\rangle\left\langle\psi_{r}^{\prime \prime}\right|=U \Pi_{r} U^{\dagger}$ for all $r$; hence, $\Pi_{r}$ and $\Pi_{r}^{\prime}$ are unitarily equivalent.

We now turn to the order-3 angle tensors. We have

Theorem 3: Let $\theta_{r s t}$ be a real completely antisymmetric tensor. Then the following conditions are equivalent.

1. $\theta_{r s t}$ is the order-3 angle tensor for a SIC-family.

2. For some fixed $a$ and all $r, s, t$

$$
\theta_{\text {ars }}+\theta_{\text {ast }}+\theta_{\text {atr }}=\theta_{\text {rst }}
$$

and for all $r, s$

$$
\sum_{t} K_{r t} K_{t s} e^{i \theta_{r s t}}=d K_{r s}
$$

3. For some fixed $a$ and all $r, s, t$,

$$
\begin{aligned}
\theta_{\text {ars }}+\theta_{\text {ast }}+\theta_{\text {atr }} & =\theta_{r s t}, \\
\sum_{r, s, t} K_{r s} K_{s t} K_{t r} e^{i \theta_{r s t}} & =d^{4}, \\
\sum_{r, s, t, u} K_{r s} K_{s t} K_{t u} K_{u r} e^{i\left(\theta_{r s t}+\theta_{t u r}\right)} & =d^{5} .
\end{aligned}
$$

Let $\Pi_{r}$ and $\Pi_{r}^{\prime}$ be two different SIC-sets and let $\theta_{r s t}$ and $\theta_{r s t}^{\prime}$ be the corresponding order-3 angle tensors. Then the necessary and sufficient condition for there to exist a unitary $U$ such that

$$
\Pi_{r}^{\prime}=U \Pi_{r} U^{\dagger}
$$

for all $r$ is that $\theta_{r s t}^{\prime}=\theta_{r s t}$ for all $r, s, t$ (in other words two SIC-sets are unitarily equivalent if and only if their order- 3 angle tensors are identical).

Let $\theta_{r s t}$ be the order-3 angle tensor corresponding to a SIC-family. Then the order-2 angle tensor is given by (up to gauge freedom)

$$
\theta_{r s}=\theta_{\text {ars }}
$$

for any fixed a, from which the SIC-family can be reconstructed using the method described in Theorem 1.

Remark: Unlike the order-2 tensor, the order-3 angle tensor is gauge invariant. This means that it provides what is, in many ways, a more useful characterization of the SIC-family. For that reason we will be almost exclusively concerned with the order- 3 tensor in the remainder of this paper. 
Proof: The fact that $(1) \Rightarrow(2)$ is an immediate consequence of the definition of the order-3 angle tensor and condition (2) of Corollary 2 . To prove that (2) $\Rightarrow$ (1) let $\theta_{r s t}$ be a completely antisymmetric tensor such that condition (2) holds. Define

$$
\theta_{r s}=\theta_{\text {ars }}
$$

for all $r, s$. Then Eq. (58) implies

$$
\sum_{t} K_{r t} K_{t s} e^{i\left(\theta_{r t}+\theta_{t s}\right)}=e^{i \theta_{r s}}\left(\sum_{t} K_{r t} K_{t s} e^{i \theta_{r s t}}\right)^{*}=d K_{r s} e^{i \theta_{r s}}
$$

for all $r, s$. It follows from Corollary 2 that $\theta_{r s}$ is the order- 2 tensor and $\theta_{r s t}$ is the order-3 angle tensor of a SIC-family.

The equivalence of conditions (1) and (3) is proved similarly.

It remains to show that two SIC-sets are unitarily equivalent if and only if their order-3 angle tensors are identical. To see this let $\Pi_{r}=\left|\psi_{r}\right\rangle\left\langle\psi_{r}\right|$ and $\Pi_{r}^{\prime}=\left|\psi_{r}^{\prime}\right\rangle\left\langle\psi_{r}^{\prime}\right|$ be two different SIC-sets having the same order-3 angle tensor $\theta_{r s t}$. Let $\theta_{r s}$ (respectively, $\theta_{r s}^{\prime}$ ) be the order-2 angle tensor corresponding to the vectors $\left|\psi_{r}\right\rangle$ (respectively, $\left|\psi_{r}^{\prime}\right\rangle$ ). Choose some fixed index $a$. We have

$$
\theta_{a r}^{\prime}+\theta_{s a}^{\prime}+\theta_{r s}^{\prime}=\theta_{a r}+\theta_{s a}+\theta_{r s}
$$

for all $r, s$. Consequently, $\theta_{r s}^{\prime}=\theta_{r s}+\phi_{r}-\phi_{s}$ for all $r, s$, where $\phi_{r}=\theta_{a r}-\theta_{a r}^{\prime}$. So $\theta_{r s}^{\prime}$ and $\theta_{r s}$ are gauge equivalent. It follows from Corollary 2 that $\Pi_{r}$ and $\Pi_{r}^{\prime}$ are unitarily equivalent.

Conversely, suppose that $\Pi_{r}$ and $\Pi_{r}^{\prime}$ are unitarily equivalent and let $\theta_{r s}$ and $\theta_{r s}^{\prime}$ be order-2 angle tensors corresponding to them. It follows from Corollary 2 that $\theta_{r s}$ and $\theta_{r s}^{\prime}$ are gauge equivalent. It is then immediate that the order- 3 angle tensors are identical.

Finally, let us note that when expressed in terms of the triple products, Eq. (58) reads

$$
\sum_{t} T_{r s t}=d K_{r s}^{2}
$$

while Eq. (60) reads

$$
\sum_{r, s, t} T_{r s t}=d^{4}
$$

For Eq. (61) we have to work a little harder. We have

$$
\sum_{r, s, t, u} \frac{1}{K_{r t}^{2}} T_{r s t} T_{t u r}=d^{5}
$$

from which it follows:

$$
d^{5}=\sum_{r, s, t, u}\left(-d \delta_{r t}+d+1\right) T_{r s t} T_{t u r}=(d+1) \sum_{r, s, t, u} T_{r s t} T_{t u r}-d^{5} .
$$

Consequently,

$$
\sum_{s, u} \operatorname{Tr}\left(T_{s} T_{u}\right)=\sum_{r, s, t, u} T_{r s t} T_{t u r}=\frac{2 d^{5}}{d+1} .
$$

This equation can be alternatively written

$$
\sum_{r, s} \operatorname{Tr}\left(T_{r} T_{s}\right)=\frac{2 d^{5}}{d+1},
$$

where $T_{r}$ is the matrix with matrix elements $\left(T_{r}\right)_{u v}=T_{r u v}$.

When they are written like this, in terms of the triple products, the fact that Eq. (67) implies Eqs. (68) and (71) becomes almost obvious. The reverse implication, by contrast, is rather less obvious. 


\section{SPECTRAL DECOMPOSITIONS}

Let $T_{r}, J_{r}$, and $R_{r}$ be the $d^{2} \times d^{2}$ matrices whose matrix elements are

$$
\left(T_{r}\right)_{s t}=T_{r s t} \quad\left(J_{r}\right)_{s t}=J_{r s t} \quad\left(R_{r}\right)_{s t}=R_{r s t},
$$

where $J_{r s t}$ and $R_{r s t}$ are the quantities defined by Eqs. (12) and (13). So $J_{r}$ is the adjoint representation matrix of $\Pi_{r}$. In this section we derive the spectral decompositions of these matrices. To avoid confusion we will use the notation $|\psi\rangle$ to denote a ket in $d$ dimensional Hilbert space $\mathcal{H}_{d}$ and $\| \psi\rangle\rangle$ to denote a ket in $d^{2}$ dimensional Hilbert space $\mathcal{H}_{d^{2}}$. In terms of this notation the spectral decompositions will turn out to be,

$$
\begin{gathered}
\left.\left.T_{r}=\frac{d}{d+1} Q_{r}+\frac{2 d}{d+1} \| e_{r}\right\rangle\right\rangle\left\langle\left\langle e_{r} \|,\right.\right. \\
J_{r}=Q_{r}-Q_{r}^{\mathrm{T}}, \\
\left.\left.R_{r}=Q_{r}+Q_{r}^{\mathrm{T}}+4 \| e_{r}\right\rangle\right\rangle\left\langle\left\langle e_{r} \| .\right.\right.
\end{gathered}
$$

In these expressions the vector $\left.\left.\| e_{r}\right\rangle\right\rangle$ is normalized, and its components in the standard basis are all real. $Q_{r}$ is a rank $d-1$ projector such that

$$
\left.\left.\left.\left.Q_{r} \| e_{r}\right\rangle\right\rangle=Q_{r}^{\mathrm{T}} \| e_{r}\right\rangle\right\rangle=0
$$

and which has, in addition, the remarkable property of being orthogonal to its own transpose (also a rank $d-1$ projector),

$$
Q_{r} Q_{r}^{\mathrm{T}}=0 .
$$

Explicit expressions for $\left.\left.\| e_{r}\right\rangle\right\rangle$ and $Q_{r}$ will be given below.

It will be convenient to define the rank $2(d-1)$ projector

$$
\bar{R}_{r}=Q_{r}+Q_{r}^{\mathrm{T}}
$$

We have

$$
\begin{aligned}
& \bar{R}_{r}=J_{r}^{2}, \\
& \left.\left.R_{r}=\bar{R}_{r}+4 \| e_{r}\right\rangle\right\rangle\left\langle\left\langle e_{r} \| .\right.\right.
\end{aligned}
$$

Since $Q_{r}$ is a Hermitian we have

$$
Q_{r}^{\mathrm{T}}=Q_{r}^{*},
$$

where $Q_{r}^{*}$ is the matrix whose elements are the complex conjugates of the corresponding elements of $Q_{r}$. So $\bar{R}_{r}$ is twice the real part of $Q_{r}$ and $-i J_{r}$ is twice the imaginary part.

In Sec. VI we will show that Eq. (75) is essentially definitive of a SIC-POVM. To be more specific, let $L_{r}$ be any set of $d^{2}$ Hermitian matrices which constitute a basis for $\operatorname{gl}(d, \mathbb{C})$, and let $C_{r}$ be the adjoint representative of $L_{r}$ in that basis. Then we will show that $C_{r}$ has the spectral decomposition

$$
C_{r}=Q_{r}-Q_{r}^{\mathrm{T}},
$$

where $Q_{r}$ is a rank $d-1$ projector which is orthogonal to its own transpose if and only if the $L_{r}$ are a family of SIC-projectors up to multiplication by a sign and shifting by a multiple of the identity.

Having stated our results let us now turn to the task of proving them. We begin by deriving the spectral decomposition of $T_{r}$. Multiplying both sides of the equation,

$$
\Pi_{r} \Pi_{s}=\frac{d+1}{d} \sum_{t} T_{r s t} \Pi_{t}-K_{r s}^{2} I
$$


by $\Pi_{r}$ we find

$$
\Pi_{r} \Pi_{s}=\frac{(d+1)^{2}}{d^{2}} \sum_{t}\left(T_{r}\right)_{s t}^{2} \Pi_{t}-\frac{d+1}{d} \sum_{t} T_{r s t} K_{r t}^{2} I-K_{r s}^{2} \Pi_{r} .
$$

Then some elementary algebra shows that

$$
\sum_{t} T_{r s t} K_{r t}^{2}=\frac{2 d}{d+1} K_{r s}^{2}
$$

and hence

$$
\Pi_{r} \Pi_{s}=\frac{d+1}{d} \sum_{t}\left(\frac{d+1}{d}\left(T_{r}\right)_{s t}^{2}-K_{r s}^{2} K_{r t}^{2}\right) \Pi_{t}-K_{r s}^{2} I .
$$

Comparing with Eq. (84) we deduce

$$
\left(T_{r}\right)_{s t}^{2}=\frac{d}{d+1} T_{r s t}+\frac{d}{d+1} K_{r s}^{2} K_{r t}^{2}
$$

Now define

$$
\left.\left.\left.\left.\| e_{r}\right\rangle\right\rangle=\sqrt{\frac{d+1}{2 d}} \sum_{s} K_{r s}^{2} \| s\right\rangle\right\rangle
$$

where the basis kets $\| s\rangle\rangle$ are given by (in column vector form)

$$
\left.\left.\left.\left.\| 1\rangle\rangle=\left(\begin{array}{c}
1 \\
0 \\
\vdots \\
0
\end{array}\right), \| 2\right\rangle\right\rangle=\left(\begin{array}{c}
0 \\
1 \\
\vdots \\
0
\end{array}\right), \ldots, \| d^{2}\right\rangle\right\rangle=\left(\begin{array}{c}
0 \\
0 \\
\vdots \\
1
\end{array}\right) .
$$

It is easily verified that $\left.\left.\| e_{r}\right\rangle\right\rangle$ is normalized. Equation (88) then becomes

$$
\left.\left.T_{r}^{2}=\frac{d}{d+1} T_{r}+\frac{2 d^{2}}{(d+1)^{2}} \| e_{r}\right\rangle\right\rangle\left\langle\left\langle e_{r} \|\right.\right.
$$

Using Eq. (86) and some simple algebra we find

$$
\left\langle\left\langle s\left\|T_{r}\right\| e_{r}\right\rangle\right\rangle=\frac{2 d}{d+1}\left\langle\left\langle s \| e_{r}\right\rangle\right\rangle,
$$

so $\left.\left.\| e_{r}\right\rangle\right\rangle$ is an eigenvector of $T_{r}$ with eigenvalue $\frac{2 d}{d+1}$.

Also define

$$
\left.\left.Q_{r}=\frac{d+1}{d} T_{r}-2 \| e_{r}\right\rangle\right\rangle\left\langle\left\langle e_{r} \|\right.\right.
$$

So in terms of the order-3 angle tensor the matrix elements of $Q_{r}$ are

$$
Q_{r s t}=\frac{d+1}{d} K_{r s} K_{r t}\left(K_{s t} e^{i \theta_{r s t}}-K_{r s} K_{r t}\right) \text {. }
$$

$Q_{r}$ is Hermitian (because $T_{r}$ is Hermitian) and is easily verified to be idempotent; hence, it is a projection operator. That $Q_{r}$ has rank $d-1$ follows from taking the trace of $T_{r}$.

We have thus proved that the spectral decomposition of $T_{r}$ is

$$
\left.\left.T_{r}=\frac{d}{d+1} Q_{r}+\frac{2 d}{d+1} \| e_{r}\right\rangle\right\rangle\left\langle\left\langle e_{r} \|,\right.\right.
$$

where $Q_{r}$ is a rank $d-1$ projector, as claimed.

We next prove that $\left.\left.Q_{r}^{\mathrm{T}} \| e_{r}\right\rangle\right\rangle=0$. The fact that the components of $\left.\left.\| e_{r}\right\rangle\right\rangle$ in the standard basis are all real means

$$
\left\langle\left\langle s\left\|T_{r}^{\mathrm{T}}\right\| e_{r}\right\rangle\right\rangle=\left\langle\left\langle e_{r}\left\|T_{r}\right\| s\right\rangle\right\rangle=\frac{2 d}{d+1}\left\langle\left\langle s \| e_{r}\right\rangle\right\rangle .
$$


So $\left.\| e_{r}\right\rangle$ is an eigenvector of $T_{r}^{\mathrm{T}}$ as well as $T_{r}$, again with the eigenvalue $\frac{2 d}{d+1}$. In view of Eq. (93) it follows that $\left.\left.Q_{r}^{\mathrm{T}} \| e_{r}\right\rangle\right\rangle=0$.

Turning to the problem of showing that $Q_{r}$ is orthogonal to its own transpose, we have

$$
\left.\left.Q_{r} Q_{r}^{\mathrm{T}}=\frac{(d+1)^{2}}{d^{2}} T_{r} T_{r}^{\mathrm{T}}-4 \| e_{r}\right\rangle\right\rangle\left\langle\left\langle e_{r} \| .\right.\right.
$$

It follows from Eq. (24) that

$$
\left\langle\left\langle s\left\|T_{r} T_{r}^{\mathrm{T}}\right\| t\right\rangle\right\rangle=\sum_{u} T_{r s u} T_{r t u}=G_{r s} G_{r t} \sum_{u} G_{s u} G_{t u} G_{u r} G_{u r} .
$$

In view of Eq. (23) (i.e., the fact that every SIC-POVM is a 2-design) this implies

$$
\left\langle\left\langle s\left\|T_{r} T_{r}^{\mathrm{T}}\right\| t\right\rangle\right\rangle=\frac{2 d}{d+1}\left|G_{r s}\right|^{2}\left|G_{r t}\right|^{2}=\frac{4 d^{2}}{(d+1)^{2}}\left\langle\left\langle s \| e_{r}\right\rangle\right\rangle\left\langle\left\langle e_{r} \| t\right\rangle\right\rangle,
$$

which gives us an expression for $T_{r} T_{r}^{\mathrm{T}}$ and we conclude that $Q_{r} Q_{r}^{\mathrm{T}}=0$.

Equations (75) and (76) are immediate consequences of the results already proved and the definitions of $J_{r}$ and $R_{r}$.

We defined the $J$ matrices to be the adjoint representatives of the SIC-projectors, considered as a basis for the Lie algebra $\operatorname{gl}(d, \mathbb{C})$, and that is certainly a most important fact about them. However, the results of this section show that, along with the vectors $\left.\left.\| e_{r}\right\rangle\right\rangle$, they actually determine the whole structure. Specifically, we have

$$
\begin{aligned}
& Q_{r}=\frac{1}{2}\left(J_{r}+J_{r}^{2}\right), \\
& \left.\left.R_{r}=J_{r}^{2}+4 \| e_{r}\right\rangle\right\rangle\left\langle\left\langle e_{r} \|,\right.\right. \\
& \left.T_{r}=\frac{d}{2(d+1)}\left(J_{r}+J_{r}^{2}+4 \| e_{r}\right\rangle\right\rangle\left\langle\left\langle e_{r} \|\right) .\right.
\end{aligned}
$$

Moreover, if we know the $T$ matrices then we know the order- 3 angle tensor, which in view of Theorem 3 means we can reconstruct the SIC-projectors. Since the vectors $\left.\left.\| e_{r}\right\rangle\right)$ are given, once and for all, this means that the problem of proving the existence of a SIC-POVM in dimension $d$ is equivalent to the problem of proving the existence of a certain remarkable structure in the adjoint representation of $\operatorname{gl}(d, \mathbb{C}$ ) (as we will see in more detail in Sec. VI).

In Sec. I we began with the concept of a SIC-POVM and then defined the $J$ matrices in terms of it. However, one could, if one wished, go in the opposite direction and take the Lie algebraic structure to be primary, with the SIC-POVM being the secondary, derivative entity.

\section{THE $Q-Q^{\top}$ PROPERTY}

Sections V-IX are devoted to a study of the $J$ matrices which, as we will see, have numerous interesting properties. We begin our investigation by trying to get some additional insight into what we will call the $Q-Q^{\mathrm{T}}$ property: namely, the fact that the $J$ matrices have the spectral decomposition

$$
J_{r}=Q_{r}-Q_{r}^{\mathrm{T}},
$$

where $Q_{r}$ is a rank $d-1$ projector which is orthogonal to its own transpose. We wish to characterize the general class of matrices which are of this type. The following theorem provides one such characterization.

Theorem 4: Let A be a Hermitian matrix. Then the following statements are equivalent.

1. A has the spectral decomposition

$$
A=P-P^{\mathrm{T}},
$$

where $P$ is a projector which is orthogonal to its own transpose. 
2. A is pure imaginary and $A^{2}$ is a projector.

Proof: To show that $(1) \Rightarrow(2)$ observe that the fact that $P$ is Hermitian means $P^{\mathrm{T}}=P^{*}$, where $P^{*}$ is the matrix whose elements are the complex conjugates of the corresponding elements of $P$. So Eq. (104) implies that the components of $A$ are pure imaginary. Since $P P^{\mathrm{T}}=0$ it also implies that $A^{2}$ is a projector.

To show that (2) $\Rightarrow(1)$ observe that the fact that $A^{2}$ is a projector means that the eigenvalues of $A= \pm 1$ or 0 . So $A=P-P^{\prime}$, where $P$ and $P^{\prime}$ are orthogonal projectors. Since $A$ is pure imaginary $A^{\mathrm{T}}=A^{*}=-A$. Now, $P^{\mathrm{T}}$ and $\left(P^{\prime}\right)^{\mathrm{T}}$ are also orthogonal projectors. So if $P^{\mathrm{T}}|\psi\rangle=|\psi\rangle$ and $|\psi\rangle$ is normalized, we must have

$$
1=\left\langle\psi\left|P^{\mathrm{T}}\right| \psi\right\rangle=\left\langle\psi\left|\left(P^{\mathrm{T}}-\left(P^{\prime}\right)^{\mathrm{T}}\right)\right| \psi\right\rangle=\left\langle\psi\left|P^{\prime}\right| \psi\right\rangle-\langle\psi|P| \psi\rangle .
$$

Since $0 \leq\langle\psi|P| \psi\rangle \leq 1$, and similarly for $P^{\prime}$, we must have $\left\langle\psi\left|P^{\prime}\right| \psi\right\rangle=1$, implying $P^{\prime}|\psi\rangle=|\psi\rangle$. Similarly $P^{\prime}|\psi\rangle=|\psi\rangle$ implies $P^{\mathrm{T}}|\psi\rangle=|\psi\rangle$. Therefore $P^{\prime}=P^{\mathrm{T}}$.

We also have the following statement, inspired in part by Ref. 51 .

Theorem 5: The necessary and sufficient condition for a matrix $P$ to be a projector which is orthogonal to its own transpose is that

$$
P=S D S^{\mathrm{T}}
$$

where $S$ is an any real orthogonal matrix and $D$ has the block-diagonal form

$$
D=\bigoplus_{j=1}^{n} \sigma
$$

with

$$
\sigma=\frac{1}{2}\left(\begin{array}{ll}
1 & -i \\
i & 1
\end{array}\right)
$$

In other words $D$ has $n$ copies of $\sigma$ on the diagonal, where $n=\operatorname{rank}(P)$, and 0 everywhere else.

Proof: Sufficiency is an immediate consequence of the fact that $\sigma$ is a rank-1 projector such that $\sigma \sigma^{\mathrm{T}}=0$.

To prove necessity let $d$ be the dimension of the space and $n$ be the rank of $P$, and let $P_{r s}$ be the matrix elements of $P$ in the canonical basis. Now let $\left|a_{1}\right\rangle, \ldots,\left|a_{n}\right\rangle$ be an orthonormal basis for the subspace onto which $P$ projects and let $\left|a_{r}^{*}\right\rangle$ be the column vector which is obtained from $\left|a_{r}\right\rangle$ by taking the complex conjugate of each of its components. Taking complex conjugates on each side of the equation $P\left|a_{r}\right\rangle=\left|a_{r}\right\rangle$ gives $P^{*}\left|a_{r}^{*}\right\rangle=\left|a_{r}^{*}\right\rangle$. So $\left|a_{1}^{*}\right\rangle, \ldots,\left|a_{n}^{*}\right\rangle$ is an orthonormal basis for the subspace onto which $P^{\mathrm{T}}=P^{*}$ projects. Since $P^{\mathrm{T}}$ is orthogonal to $P$ we conclude that $\left\langle a_{r} \mid a_{s}^{*}\right\rangle=0$ for all $r, s$.

Next define vectors $\left|b_{1}\right\rangle, \ldots,\left|b_{2 n}\right\rangle$ by

$$
\left|b_{2 r-1}\right\rangle=\frac{1}{\sqrt{2}}\left(\left|a_{r}^{*}\right\rangle-\left|a_{r}\right\rangle\right), \quad\left|b_{2 r}\right\rangle=\frac{i}{\sqrt{2}}\left(\left|a_{r}^{*}\right\rangle+\left|a_{r}\right\rangle\right) .
$$

By construction these vectors are orthonormal and real. So we can extend them to an orthonormal basis for the full space by adding a further $d-2 n$ vectors $\left|b_{2 n+1}\right\rangle, \ldots,\left|b_{d}\right\rangle$, which can also be chosen to be real. We have

$$
P=\sum_{r=1}^{n}\left|a_{r}\right\rangle\left\langle a_{r}\right|=\frac{1}{2} \sum_{r=1}^{n}\left(\left|b_{2 r-1}\right\rangle\left\langle b_{2 r-1}|-i| b_{2 r-1}\right\rangle\left\langle b_{2 r}|+i| b_{2 r}\right\rangle\left\langle b_{2 r-1}|+| b_{2 r}\right\rangle\left\langle b_{2 r}\right|\right) .
$$

So if we define

$$
S=\sum_{r=1}^{d}\left|b_{r}\right\rangle\langle r|,
$$

then $S$ is a real orthogonal matrix such that $P=S D S^{\mathrm{T}}$. 
This result implies the following alternative characterization of the class of matrices to which the $J$ matrices belong.

Corollary 6: Let A be a Hermitian matrix. Then the following statements are equivalent.

1. A has the spectral decomposition

$$
A=P-P^{\mathrm{T}},
$$

where $P$ is a projector which is orthogonal to its own transpose.

2. There exists a real orthogonal matrix $S$ such that

$$
A=S D S^{\mathrm{T}},
$$

where $D$ has the block-diagonal form

$$
D=\bigoplus_{j=1}^{n} \sigma_{y}
$$

with $\sigma_{y}$ being the Pauli matrix

$$
\sigma_{y}=\left(\begin{array}{ll}
0 & -i \\
i & 0
\end{array}\right)
$$

In other words $D$ has $n$ copies of $\sigma_{y}$ on the diagonal, where $n=\frac{1}{2} \operatorname{rank}(A)$, and 0 every where else (note that a matrix of this type must have even rank).

Proof: Immediate consequence of Theorem 5.

\section{LIE ALGEBRAIC FORMULATION OF THE EXISTENCE PROBLEM}

This section is the core of the paper. We show that the problem of proving the existence of a SIC-POVM in dimension $d$ is equivalent to the problem of proving the existence of a Hermitian basis for $\operatorname{gl}(d, \mathbb{C})$; all of whose elements have the $Q-Q^{\mathrm{T}}$ property. The main result is the following.

Theorem 7: Let $L_{r}$ be a set of $d^{2}$ Hermitian matrices forming a basis for $\operatorname{gl}(d, \mathbb{C})$. Let $C_{r s t}$ be the structure constants relative to this basis, so that

$$
\left[L_{r}, L_{s}\right]=\sum_{t} C_{r s t} L_{t},
$$

and let $C_{r}$ be the matrix with matrix elements $\left(C_{r}\right)_{s t}=C_{r s t}$. Then the following statements are equivalent.

1. Each $C_{r}$ has the spectral decomposition

$$
C_{r}=P_{r}-P_{r}^{\mathrm{T}},
$$

where $P_{r}$ is a rank $d-1$ projector which is orthogonal to its own transpose.

2. There exists a SIC-set $\Pi_{r}$, a set of signs $\epsilon_{r}= \pm 1$ and a real constant $\alpha \neq-\frac{1}{d}$ such that

$$
L_{r}=\epsilon_{r}\left(\Pi_{r}+\alpha I\right) \text {. }
$$

Remark: The restriction to values of $\alpha \neq-\frac{1}{d}$ is needed to ensure that the matrices $L_{r}$ are linearly independent, and therefore constitute a basis for $\mathrm{gl}(d, \mathbb{C})$ (otherwise they would all have zero trace). The $Q-Q^{\mathrm{T}}$ property continues to hold even if $\alpha$ does equal $-1 / d$.

It will be seen that it is not only SIC-sets which have the $Q-Q^{\mathrm{T}}$ property but also any set of operators obtained from a SIC-set by shifting by a constant and multiplying by an $r$-dependent sign. 
So the $Q-Q^{\mathrm{T}}$ property is not strictly equivalent to the property of being a SIC-set; however, it could be said that the properties are almost equivalent. In particular, the existence of a Hermitian basis for $\mathrm{gl}(d, \mathbb{C})$ having the $Q-Q^{\mathrm{T}}$ property implies the existence of a SIC-POVM in dimension $d$, and conversely.

\section{A. Proof that $(2) \Longrightarrow(1)$}

Taking the trace on both sides of

$$
\left[\Pi_{r}, \Pi_{s}\right]=\sum_{t} J_{r s t} \Pi_{t},
$$

we deduce that summing $J_{r s t}$ over all values of $t$ mush vanish. Then from the definition of $L_{r}$ in terms of $\Pi_{r}$ we find that $C_{r s t}=\epsilon_{r} \epsilon_{s} \epsilon_{t} J_{r s t}$. Consequently, $C_{r}=P_{r}-P_{r}^{\mathrm{T}}$, where $P_{r}=\epsilon_{r} S Q_{r} S$ and with $S$ being the symmetric orthogonal matrix with the sign $\epsilon_{r}$ at the $r$ th position on the diagonal. The claim is now immediate.

\section{B. Proof that $(1) \Longrightarrow(2)$}

For this we need to work harder. Since the proof is rather lengthy we will break it into a number of lemmas. We first collect a few elementary facts which will be needed in the sequel.

Lemma 8: Let $L_{r}$ be any Hermitian basis for $\mathrm{gl}(d, \mathbb{C})$, and let $C_{r s t}$ and $C_{r}$ be the structure constants and adjoint representatives as defined in the statement of Theorem 7. Let $l_{r}=\operatorname{Tr}\left(L_{r}\right)$. Then we have the following.

1. The $l_{r}$ are not all zero.

2. The $C_{r s t}$ are pure imaginary and antisymmetric in the first pair of indices.

3. The $C_{r s t}$ are completely antisymmetric if and only if the $C_{r}$ are Hermitian.

4. In every case

$$
\sum_{t} C_{r s t} l_{t}=0
$$

for all $r, s$.

5. In the special case that the $C_{r}$ are Hermitian,

$$
\sum_{r} l_{r} L_{r}=\kappa I
$$

where $\kappa>0$ and is given by $\kappa=\frac{1}{d} \sum_{r} l_{r}^{2}$.

Proof: To prove statement (1) observe that if the $l_{r}$ were all zero it would mean that the identity was not in the span of the $L_{r}$ - contrary to the assumption that they form a basis.

To prove statement(2) observe that taking Hermitian conjugates on both sides of Eq. (116) gives

$$
-\left[L_{r}, L_{s}\right]=\sum_{t} C_{r s t}^{*} L_{t}
$$

from which it follows that $C_{r s t}^{*}=-C_{r s t}$. The fact that $C_{s r t}=-C_{r s t}$ is an immediate consequence of the definition.

Statement (3) is now immediate.

Statement (4) is proved by tracing Eq. (119).

To prove statement (5) observe that if the $C_{r}$ are Hermitian it follows from statements (2) and (3) that

$$
\sum_{r} l_{r} C_{r s t}=0
$$


for all $s, t$. Consequently, the matrix $\sum_{r} l_{r} L_{r}$ commutes with everything. But the only matrices for which that is true are multiples of the identity, and it follows that $\sum_{r} l_{r} L_{r}=\kappa I$ for some real $\kappa$. Taking the trace on both sides of this equation we deduce $\sum_{r} l_{r}^{2}=d \kappa$. The fact that $\kappa>0$ is a consequence of this and statement (1).

We next observe that if the $C_{r}$ have the $Q-Q^{\mathrm{T}}$ property they must, in particular, be Hermitian. It turns out that that is, by itself, already a very strong constraint.

Before stating the result, we will explain the essential idea on which it depends. Although we have not done so before, and will not do so again, it will be convenient to make use of the covariant/contravariant index notation which is often used to describe the structure constants. Define the metric tensor $M_{r s}=\operatorname{Tr}\left(L_{r} L_{s}\right)$ and let $M^{r s}$ be its inverse. Then

$$
\sum_{t} M^{r t} M_{t s}=M_{s}^{r}= \begin{cases}1 & \text { if } r=s \\ 0 & \text { if } r \neq s .\end{cases}
$$

We can use these tensors to raise and lower indices (we use the Hilbert-Schmidt inner product for this purpose because the fact that $\operatorname{gl}(d, \mathbb{C})$ is not semisimple means that its Killing form is degenerate $\left.{ }^{52-55}\right)$. In particular, the matrices

$$
L^{r}=\sum_{t} M^{r s} L_{s}
$$

are the basis dual to the $L_{r}$, meaning $\operatorname{Tr}\left(L^{r} L_{s}\right)=M^{r}{ }_{s}$. Suppose we now define structure constants $\tilde{C}_{r s t}$ by

$$
\left[L_{r}, L_{s}\right]=\sum_{t} \tilde{C}_{r s t} L^{t}
$$

(so in terms of the $C_{r s t}$ we have $\tilde{C}_{r s}{ }^{t}=C_{r s t}$ ). It follows from the relation

$$
\tilde{C}_{r s t}=\operatorname{Tr}\left(\left[L_{r}, L_{s}\right] L_{t}\right)=\operatorname{Tr}\left(L_{r}\left[L_{s}, L_{t}\right]\right)
$$

that the $\tilde{C}_{r s t}$ are completely antisymmetric for any choice of the $L_{r}$. If we now require that the matrices $C_{r}$ be Hermitian it means that not only the $\tilde{C}_{r s t}$ but also the $C_{r s t}$ must be completely antisymmetric. Since the two quantities are related by

$$
\tilde{C}_{r s t}=\sum_{u} C_{r s u} M_{u t},
$$

this is a very strong requirement. It means that the $L_{r}$ must, in a certain sense, be close to orthonormal (relative to the Hilbert-Schmidt inner product). More precisely, it means we have the following lemma.

Lemma 9: Let $L_{r}, C_{r s t}$, and $C_{r}$ be defined as in the statement of Theorem 7 and let $l_{r}=\operatorname{Tr}\left(L_{r}\right)$. Then the $C_{r}$ are Hermitian if and only if

$$
\operatorname{Tr}\left(L_{r} L_{s}\right)=\beta \delta_{r s}+\gamma l_{r} l_{s}
$$

where $\beta$ and $\gamma$ are real constants such that $\beta>0$ and $\gamma<\frac{1}{d}$.

If this condition is satisfied we also have

$$
\begin{gathered}
\sum_{r} l_{r} L_{r}=\frac{\beta}{1-d \gamma} I, \\
\sum_{r} l_{r}^{2}=\frac{d \beta}{1-d \gamma} .
\end{gathered}
$$

Proof: To prove sufficiency observe that, in view of Eq. (128), the condition means

$$
\tilde{C}_{r s t}=\beta C_{r s t}+\gamma l_{t} \sum_{u} C_{r s u} l_{u} .
$$


From Lemma 8, and the fact that $\beta \neq 0$, this implies $C_{r s t}=\frac{1}{\beta} \tilde{C}_{r s t}$. Since the $\tilde{C}_{r s t}$ are completely antisymmetric we conclude that the $C_{r s t}$ must be also, hence the $C_{r}$ are Hermitian.

To prove necessity let $\tilde{C}_{r}$ (respectively, $M$ ) be the matrix whose matrix elements are $\tilde{C}_{r s t}$ (respectively, $M_{s t}$ ). Then Eq. (128) can be written as $\tilde{C}_{r}=C_{r} M$. Taking the transpose (or, equivalently, the Hermitian conjugate) on both sides of this equation we find $\tilde{C}_{r}=M C_{r}$, implying $\left[M, C_{r}\right]=0$ for all $r$. Since the $L_{r}$ are a basis for $\operatorname{gl}(d, \mathbb{C})$ we deduce $\left[M, \mathrm{ad}_{A}\right]=0$ for all $A \in \operatorname{gl}(d, \mathbb{C})$. Equation (129) is a straightforward consequence of this, the fact that $\operatorname{gl}(d, \mathbb{C})$ has the direct sum decomposition $\mathbb{C} I \oplus \operatorname{sl}(d, \mathbb{C})$, the fact that $\operatorname{sl}(d, \mathbb{C})$ is simple, and Schur's lemma. ${ }^{52-55}$ With similar reasoning, Eqs. (130) and (131) follow immediately from Lemma 8. We omit these details and refer the reader to the aforementioned references.

It remains to establish the bounds on $\beta$ and $\gamma$. Let $A=\sum_{r} a_{r} L_{r}$ be any nonzero element of $\operatorname{sl}(d, \mathbb{C})$. Then $\sum_{r} a_{r} l_{r}=0$, so in view of Eq. (129) we have

$$
0<\operatorname{Tr}\left(A^{2}\right)=\beta \sum_{r} a_{r}^{2} .
$$

It follows that $\beta>0$. Also, using Lemma 8 once more, we find

$$
l_{r}=\frac{1}{\kappa} \sum_{s} l_{s} \operatorname{Tr}\left(L_{r} L_{s}\right)=\frac{\beta l_{r}}{\kappa}+\frac{\gamma l_{r}}{\kappa} \sum_{s} l_{s}^{2}=l_{r}\left(\frac{\beta}{\kappa}+d \gamma\right) .
$$

Since the $l_{r}$ cannot all be zero this implies $\frac{\beta}{\kappa}=1-d \gamma$. And since $\frac{\beta}{\kappa}>0$ we have $\gamma<\frac{1}{d}$.

Equation (129) only depends on the $C_{r}$ being Hermitian. If we make the assumption that the $C_{r}$ have the $Q-Q^{\mathrm{T}}$ property we get a stronger statement.

Corollary 10: Let $L_{r}, C_{r s t}$, and $C_{r}$ be as defined in the statement of Theorem 7. Suppose that the $C_{r}$ have the spectral decomposition $C_{r}=P_{r}-P_{r}^{\mathrm{T}}$, where $P_{r}$ is a rank $d-1$ projector which is orthogonal to its own transpose. Then, we have the following.

1. For all $r, \operatorname{Tr}\left(L_{r}\right)=\epsilon_{r}^{\prime} l$;

2. For all $r, s$

$$
\operatorname{Tr}\left(L_{r} L_{s}\right)=\frac{d}{d+1} \delta_{r s}+\frac{\epsilon_{r}^{\prime} \epsilon_{s}^{\prime}}{d}\left(l^{2}-\frac{1}{d+1}\right)
$$

3. For some real constant $l>0$ and signs $\epsilon_{r}^{\prime}= \pm 1$,

$$
\sum_{r} \epsilon_{r}^{\prime} L_{r}=d l I
$$

Proof: The proof relies on the fact ${ }^{55}$ that the Killing form for $\mathrm{gl}(d, \mathbb{C})$ is related to the HilbertSchmidt inner product by $\operatorname{Tr}\left(\operatorname{ad}_{A} \operatorname{ad}_{B}\right)=2 d \operatorname{Tr}(A B)-2 \operatorname{Tr}(A) \operatorname{Tr}(B)$. Specializing to the case $A=$ $B=L_{r}$ and making use of the $Q-Q^{\mathrm{T}}$ property, we find $d-1=d \operatorname{Tr}\left(L_{r}^{2}\right)-l_{r}^{2}$. Using Lemma 9 we deduce

$$
l_{r}^{2}=\frac{d \beta-d+1}{1-d \gamma} .
$$

It follows that $l_{r}=\epsilon_{r}^{\prime} l$ for some real constant $l \geq 0$ and signs $\epsilon_{r}^{\prime}= \pm 1$. The fact that the $L_{r}$ are a basis for $\operatorname{gl}(d, \mathbb{C})$ means the $l_{r}$ cannot all be zero. So we must in fact have $l>0$. Using this result in Eq. (131) we find $\beta+d^{2} l^{2} \gamma=d l^{2}$, while Eq. (137) implies $d \beta+d l^{2} \gamma=d-1+l^{2}$. This gives us a pair of simultaneous equations in $\beta$ and $\gamma$. Solving them we obtain

$$
\beta=\frac{d}{d+1}, \quad \gamma=\frac{1}{d l^{2}}\left(l^{2}-\frac{1}{d+1}\right) .
$$

Substituting these expressions into Eqs. (129) and (130) we deduce Eqs. (135) and (136). 
The next lemma says each $L_{r}$ is a linear combination of a rank-1 projector and the identity.

Lemma 11: Let $L$ be any Hermitian matrix $\in \operatorname{gl}(d, \mathbb{C})$ which is not a multiple of the identity. Then $\operatorname{rank}\left(\operatorname{ad}_{L}\right) \geq 2(d-1)$. The lower bound is achieved if and only if $L$ is of the form $L=\eta I+\xi P$, where $P$ is a rank-1 projector and $\eta$ and $\xi$ are any pair of real numbers. The eigenvalues of $\operatorname{ad}_{L}$ are then $\pm \xi$ (each with multiplicity $d-1$ ) and 0 (with multiplicity $d^{2}-2 d+2$ ).

Proof: Let $\lambda_{1} \geq \lambda_{2} \geq \ldots \geq \lambda_{d}$ be the eigenvalues of $L$ arranged in decreasing order, and let $\left|b_{1}\right\rangle,\left|b_{2}\right\rangle, \ldots,\left|b_{d}\right\rangle$ be the corresponding eigenvectors. We may assume, without loss of generality, that the $\left|b_{r}\right\rangle$ are orthonormal. Then $\operatorname{ad}_{L}\left(\left|b_{r}\right\rangle\left\langle b_{s}\right|\right)=\left[L,\left|b_{r}\right\rangle\left\langle b_{s}\right|\right]=\left(\lambda_{r}-\lambda_{s}\right)\left|b_{r}\right\rangle\left\langle b_{s}\right|$. So the eigenvalues of ad $\operatorname{are}_{L} \lambda_{r}-\lambda_{s}$. Since $L$ is not a multiple of the identity we must have $\lambda_{r} \neq \lambda_{r+1}$ for some $r$ in the range $1 \leq r \leq d-1$. We then have that $\lambda_{s}-\lambda_{t} \neq 0$ if either $s \leq r<t$ or $t \leq r<s$. There are $2 r(d-r)$ such pairs $s, t$. So $\operatorname{rank}\left(\operatorname{ad}_{L}\right) \geq 2 r(d-r) \geq 2(d-1)$. Now suppose that the lower bound is achieved. Then $r(d-r)=d-1$, implying that $r=1$ or $d-1$. Also we must have $\lambda_{s}=\lambda_{s+1}$ for all $s \neq r$. So we have either of the two possibilities, either $L=\lambda_{2} I+\left(\lambda_{1}-\lambda_{2}\right)\left|b_{1}\right\rangle\left\langle b_{1}\right|$ or $L=\lambda_{d-1} I-\left(\lambda_{d-1}-\lambda_{d}\right)\left|b_{d}\right\rangle\left\langle b_{d}\right|$. Either way $L$ and the spectrum of $\operatorname{ad}_{L}$ are as described.

The final ingredient needed to complete the proof is as follows.

Lemma 12: Let $L_{r}, C_{r s t}$, and $C_{r}$ be as defined in the statement of Theorem 7. Suppose that the $C_{r}$ have the spectral decomposition $C_{r}=P_{r}-P_{r}^{\mathrm{T}}$, where $P_{r}$ is a rank $d-1$ projector which is orthogonal to its own transpose. Let $l$ and $\epsilon_{r}^{\prime}$ be as in the statement of Corollary 10. Then there is a fixed sign $\epsilon= \pm 1$ such that

$$
\Pi_{r}=\epsilon \epsilon_{r}^{\prime} L_{r}-\frac{\epsilon l-1}{d} I
$$

is a rank-1 projector for all $r$.

Proof: Define

$$
L_{r}^{\prime}=\epsilon_{r}^{\prime} L_{r}-\frac{l-1}{d} I .
$$

Then it follows from Corollary 10 that this has unit trace for all $r$. Furthermore, we have

$$
\operatorname{Tr}\left(L_{r}^{\prime} L_{s}^{\prime}\right)=\frac{d \delta_{r s}+1}{d+1} \text { and } \sum_{r} L_{r}^{\prime}=d I .
$$

It is also easily seen that if we define $C_{r s t}^{\prime}=\epsilon_{r}^{\prime} \epsilon_{s}^{\prime} \epsilon_{t}^{\prime} C_{r s t}$, then

$$
\left[L_{r}^{\prime}, L_{s}^{\prime}\right]=\sum_{t} C_{r s t}^{\prime} L_{t}^{\prime}
$$

and $C_{r}^{\prime}=P_{r}^{\prime}-P_{r}^{\prime \text { T }}$, where $P_{r}^{\prime}$ is a rank-1 projector which is orthogonal to its own transpose (see the first part of the proof of Theorem 7). In particular, $\operatorname{rank}\left(\operatorname{ad}_{L_{r}^{\prime}}\right)=2(d-1)$, and the eigenvalues of ad $L_{L_{r}^{\prime}}$ are all equal to \pm 1 or 0 . So, taking account of the fact that $\operatorname{Tr}\left(L_{r}^{\prime}\right)=1$, we can use Lemma 11 to deduce that there is a family of rank-1 projectors $\Pi_{r}^{\prime}$ and signs $\xi_{r}= \pm 1$ such that

$$
L_{r}^{\prime}=\xi_{r} \Pi_{r}^{\prime}+\frac{1-\xi_{r}}{d} I
$$

If $\xi_{r}=+1$ (respectively, -1 ) for all $r$, then Eq. (139) holds with $\Pi_{r}=\Pi_{r}^{\prime}$ and $\epsilon=+1$ (respectively, -1 ). Also, if $d=2$ then $L_{r}^{\prime}$ is a rank-1 projector irrespective of the value of $\xi_{r}$, so Eq. (139) holds with $\Pi_{r}=L_{r}^{\prime}$ and $\epsilon=+1$. The problem therefore reduces to showing that if $d>2$ it cannot happen that $\xi_{r}=+1$ for some values of $r$ and -1 for others. We will do this by assuming the contrary and deducing a contradiction.

Let $m$ be the number of values of $r$ for which $\xi_{r}=+1$. We are assuming that $m$ is in the range $1 \leq m \leq d^{2}-1$. We may also assume, without loss of generality, that the labeling is such that 
$\xi_{r}=+1$ for the first $m$ values of $r$ and -1 for the rest. So

$$
L_{r}^{\prime}= \begin{cases}\Pi_{r}^{\prime} & \text { if } r \leq m, \\ \frac{2}{d} I-\Pi_{r}^{\prime} & \text { if } r>m .\end{cases}
$$

Now define $\tilde{T}_{r s t}=\operatorname{Tr}\left(L_{r}^{\prime} L_{s}^{\prime} L_{t}^{\prime}\right)$. Equation (141) means that the same argument which led to Eq. (9) can be used to deduce

$$
L_{r}^{\prime} L_{s}^{\prime}=\frac{d+1}{d}\left(\sum_{t} \tilde{T}_{r s t} L_{t}^{\prime}\right)-K_{r s}^{2} I .
$$

Since $L_{1}^{\prime}$ is a projector it follows that

$$
L_{1}^{\prime} L_{s}^{\prime}=\left(L_{1}^{\prime}\right)^{2} L_{s}^{\prime}=\frac{d+1}{d}\left(\sum_{t} \tilde{T}_{1 s t} L_{1}^{\prime} L_{t}^{\prime}\right)-K_{1 s}^{2} L_{1}^{\prime} .
$$

By essentially the same argument which led to Eq. (91) we can use this to infer

$$
\left.\left.\left(\tilde{T}_{1}^{\prime}\right)^{2}=\frac{d}{d+1} \tilde{T}_{1}+\frac{2 d^{2}}{(d+1)^{2}} \| e_{1}\right\rangle\right\rangle\left\langle\left\langle e_{1} \|,\right.\right.
$$

where $\tilde{T}_{1}^{\prime}$ is the matrix with matrix elements $\tilde{T}_{1 r s}^{\prime}$ and $\left.\| e_{1}\right\rangle$ is the vector defined by Eq. (89). As before $\left.\left.\| e_{1}\right\rangle\right\rangle$ is an eigenvector of $\tilde{T}_{1}^{\prime}$ with eigenvalue $\frac{2 d}{d+1}$. Consequently, the matrix

$$
\left.\left.\tilde{Q}_{1}=\frac{d+1}{d} \tilde{T}_{1}^{\prime}-2 \| e_{1}\right\rangle\right\rangle\left\langle e_{1} \|\right.
$$

is a projector. But that means $\operatorname{Tr}\left(\tilde{Q}_{1}\right)$ must be an integer. We now use this to derive a contradiction.

It follows from Eq. (144) that

$$
\left(L_{r}^{\prime}\right)^{2}= \begin{cases}L_{r}^{\prime} & r \leq m \\ \frac{2(d-2)}{d^{2}} I-\frac{d-4}{d} L_{r}^{\prime} & r>m .\end{cases}
$$

Consequently,

$$
\tilde{T}_{1 r r}= \begin{cases}K_{1 r}^{2} & r \leq m, \\ \frac{2(d-2)}{d^{2}}-\frac{d-4}{d} K_{1 r}^{2} & r>m,\end{cases}
$$

and so

$$
\operatorname{Tr}\left(\tilde{Q}_{1}\right)=\frac{d+1}{d} \sum_{r} \tilde{T}_{1 r r}-2=d+1-\frac{4 d^{2}+2 m(d-2)}{d^{3}} .
$$

So if $\operatorname{Tr}\left(\tilde{Q}_{1}\right)$ is an integer, $\left(4 d^{2}+2 n(d-2)\right) / d^{3}$ must also be an integer. But the fact that $1 \leq m<d^{2}$ together with the fact that $d>2$ means $\frac{4}{d}<\frac{4 d^{2}+2 m(d-2)}{d^{3}}<2$. If $d=3$ or 4 there are no integers in this interval, which gives us a contradiction straight away. If, on the other hand, $d \geq 5$ there is the possibility $4 d^{2}+2 m(d-2)=d^{3}$, implying

$$
m=\frac{d^{2}(d-4)}{2(d-2)} .
$$

This equation has the solution $d=6, m=9$ (this is in fact the only integer solution, as can be seen from an analysis of the possible prime factorizations of the numerator and denominator on the right hand side). To eliminate this possibility define $L_{r}^{\prime \prime}=-L_{d^{2}+1-r}^{\prime}+2 I / d$ for all $r$. The following three properties are easily verified:

$$
\operatorname{Tr}\left(L_{r}^{\prime \prime} L_{s}^{\prime \prime}\right)=\frac{d \delta_{r s}+1}{d+1}, \quad \sum_{r} L_{r}^{\prime \prime}=d I, \quad L_{r}^{\prime \prime}= \begin{cases}\Pi_{r} & \text { for } r \leq d^{2}-m, \\ \frac{2}{d} I-\Pi_{r} & \text { for } r>d^{2}-m .\end{cases}
$$

So we can go through the same argument as before to deduce

$$
d^{2}-m=\frac{d^{2}(d-4)}{2(d-2)} .
$$


Equations (152) and (154) have no joint solutions at all with $d \neq 0$, integer, or otherwise.

To complete the proof of Theorem 7 observe that Eqs. (135) and (139) imply

$$
\operatorname{Tr}\left(\Pi_{r} \Pi_{s}\right)=\frac{d \delta_{r s}+1}{d+1},
$$

so the $\Pi_{r}$ are a SIC-set. Moreover, $L_{r}=\epsilon_{r}\left(\Pi_{r}+\alpha I\right)$, where $\epsilon_{r}=\epsilon \epsilon_{r}^{\prime}$ and $\alpha=(\epsilon l-1) / d$.

\section{THE ALGEBRA $s \mid(d, \mathbb{C})$}

Thus far, we have focused our attention on $\operatorname{gl}(d, \mathbb{C})$, as that is the case where the connection between the Lie algebras and the SIC-POVMs seems most straightforward. However, it may be worth mentioning that a SIC-POVM also gives rise to an interesting geometrical structure in $\operatorname{sl}(d, \mathbb{C})$.

Let $\Pi_{r}$ be a SIC-set and define

$$
B_{r}=\sqrt{\frac{d+1}{2\left(d^{2}-1\right)}}\left(\Pi_{r}-\frac{1}{d} I\right) .
$$

Hence, $B_{r} \in \operatorname{sl}(d, \mathbb{C})$. Now let $\left\langle A, A^{\prime}\right\rangle=\operatorname{Tr}\left(\operatorname{ad}_{A} \operatorname{ad}_{A^{\prime}}\right)=2 d \operatorname{Tr}\left(A A^{\prime}\right)$ be the Killing form ${ }^{55}$ on $\mathrm{sl}(d, \mathbb{C})$. Then the $B_{r}$ are normalized $\left(\left\langle B_{r}, B_{r}\right\rangle=1\right)$ and have the overlap $\left\langle B_{r}, B_{s}\right\rangle=-\frac{1}{d^{2}-1}$ for $r \neq s$ and hence form a regular simplex in $\operatorname{sl}(d, \mathbb{C})$. Since $\operatorname{sl}(d, \mathbb{C})$ is $d^{2}-1$ dimensional, the $B_{r}$ are an overcomplete set. However, the fact that the sum over the $B_{r}$ vanishes means that for each $A \in \operatorname{sl}(d, \mathbb{C})$ there is a unique set of expansion coefficients $a_{r}$ such that $A=\sum_{r} a_{r} B_{r}$ and the $a_{r}$ sum to zero. It is easily checked that the $a_{r}$ can be calculated using $d^{2} a_{r}=\left(d^{2}-1\right)\left\langle A, B_{r}\right\rangle$. Similarly, given any linear transformation $M: \operatorname{sl}(d, \mathbb{C}) \rightarrow \operatorname{sl}(d, \mathbb{C})$, there is a unique set of numbers $M_{r s}$ such that

$$
M B_{r}=\sum_{s} M_{r s} B_{s}
$$

and summing $M_{r s}$ over either index vanishes. These $M_{r s}$ can be calculated similar to the $a_{r}$ using $d^{2} M_{r s}=\left(d^{2}-1\right)\left\langle B_{s}, M B_{r}\right\rangle$. In short, the $B_{r}$ retain many analogous properties of, and can be used in much the same way as, a basis.

\section{FURTHER IDENTITIES}

In Secs. I-VII we have seen that there are five different families of matrices naturally associated with a SIC-POVM: namely, the projectors $Q_{r}$ together with the matrices $J_{r}=Q_{r}-Q_{r}^{\mathrm{T}}, \bar{R}_{r}=$ $\left.\left.Q_{r}+Q_{r}^{\mathrm{T}}, R_{r}=Q_{r}+Q_{r}^{\mathrm{T}}+4 \| e_{r}\right\rangle\right\rangle\left\langle\left\langle e_{r} \|\right.\right.$, and

$$
\left.\left.T_{r}=\frac{d}{d+1} Q_{r}+\frac{2 d}{d+1} \| e_{r}\right\rangle\right\rangle\left\langle e_{r} \|\right.
$$

(see Sec. IV). As we noted previously, it is possible to define everything in terms of the adjoint representation matrices $J_{r}$ and the rank-1 projectors $\left.\left.\| e_{r}\right\rangle\right\rangle\left\langle e_{r} \|\right.$, so that $Q_{r}=\frac{1}{2} J_{r}\left(J_{r}+I\right), \bar{R}_{r}=J_{r}^{2}$, $\left.\left.R_{r}=J_{r}^{2}+4 \| e_{r}\right\rangle\right\rangle\left\langle\left\langle e_{r} \|\right.\right.$, and

$$
\left.\left.T_{r}=\frac{d}{2(d+1)} J_{r}\left(J_{r}+I\right)+\frac{2 d}{d+1} \| e_{r}\right\rangle\right\rangle\left\langle\left\langle e_{r} \| .\right.\right.
$$

In that sense the structure constants of the Lie algebra, supplemented with the vectors $\left.\left.\| e_{r}\right\rangle\right\rangle$, determine everything else.

In Sec. IX we will show that there are some interesting geometrical relationships between the hyperplanes onto which $Q_{r}, Q_{r}^{\mathrm{T}}$, and $\bar{R}_{r}$ project. In this section, as a preliminary to that investigation, we prove a number of identities satisfied by the $Q, J$, and $\bar{R}$ matrices. We start by computing their Hilbert-Schmidt inner products. 
Theorem 13: For all $r, s$ we have the following inner products:

$$
\begin{gathered}
\operatorname{Tr}\left(Q_{r} Q_{s}\right)=\frac{d^{3} \delta_{r s}+d^{2}-d-1}{(d+1)^{2}}, \quad \operatorname{Tr}\left(Q_{r} Q_{s}^{\mathrm{T}}\right)=\frac{d^{2}\left(1-\delta_{r s}\right)}{(d+1)^{2}}, \\
\operatorname{Tr}\left(J_{r} J_{s}\right)=\frac{2\left(d^{2} \delta_{r s}-1\right)}{d+1}, \quad \operatorname{Tr}\left(\bar{R}_{r} \bar{R}_{s}\right)=\frac{2(d-1)\left(d^{2} \delta_{r s}+2 d+1\right)}{(d+1)^{2}}, \quad \operatorname{Tr}\left(J_{r} \bar{R}_{s}\right) s=0 .
\end{gathered}
$$

Proof: Let us first calculate some auxiliary quantities. It follows from the definition of $T_{r}$ that the matrix $P=\frac{1}{d} G$ defined by Eq. (48) is a rank- $d$ projector and Eq. (23) (i.e., the fact that every SIC-POVM is a 2-design) that

$$
\operatorname{Tr}\left(T_{r} T_{s}\right)=\frac{d^{2}\left(d(d+2) \delta_{r s}+2 d+3\right)}{(d+1)^{3}} \quad \text { and } \quad \operatorname{Tr}\left(T_{r} T_{s}^{\mathrm{T}}\right)=\frac{2 d^{2}\left(d \delta_{r s}+d+2\right)}{(d+1)^{3}} .
$$

Next, we have the following straightforward consequences of the definitions of $T_{r}, T_{r}^{\mathrm{T}}$, and $\left.\left.\| e_{r}\right\rangle\right\rangle$ :

$$
\left\langle\left\langle e_{r}\left\|T_{s}\right\| e_{r}\right\rangle\right\rangle=\left\langle\left\langle e_{r}\left\|T_{s}^{\mathrm{T}}\right\| e_{r}\right\rangle\right\rangle=\frac{d\left(3 d \delta_{r s}+d+4\right)}{2(d+1)^{2}} \quad \text { and } \quad\left\langle\left\langle e_{r} \| e_{s}\right\rangle\right\rangle=\frac{d \delta_{r s}+d+2}{2(d+1)} .
$$

Using these results and using the expressions for $Q_{r}$ and $Q_{r}^{\mathrm{T}}$ in terms of $T_{r}$ and $\left.\| e_{r}\right\rangle$ yields the first two statements. The remaining statements are immediate consequences of these and the fact that $J_{r}=Q_{r}-Q_{r}^{\mathrm{T}}$ and $\bar{R}_{r}=Q_{r}+Q_{r}^{\mathrm{T}}$.

Now define

$$
\left.\left.\left.\left.\| v_{0}\right\rangle\right\rangle=\frac{1}{d} \sum_{r} \| r\right\rangle\right\rangle
$$

where $\| r\rangle\rangle$ is the basis defined in Eq. (90). The following result shows (among other things) that the subspaces onto which the $Q_{r}$ (respectively, $Q_{r}^{\mathrm{T}}$ and $R_{r}$ ) project span the orthogonal complement of $\left.\| v_{0}\right\rangle$.

Theorem 14: For all $r$

$$
\left.\left.\left.\left.\left.\left.\left.\left.Q_{r} \| v_{0}\right\rangle\right\rangle=Q_{r}^{\mathrm{T}} \| v_{0}\right\rangle\right\rangle=J_{r} \| v_{0}\right\rangle\right\rangle=R_{r} \| v_{0}\right\rangle\right\rangle=0 .
$$

Moreover, the sum of the $J_{r}$ vanishes, and we have

$$
\begin{array}{r}
\left.\sum_{r} Q_{r}=\sum_{r} Q_{r}^{\mathrm{T}}=\frac{d^{2}}{d+1}\left(I-\| v_{0}\right\rangle\right\rangle\left\langle\left\langle v_{0} \|\right),\right. \\
\left.\sum_{r} \bar{R}_{r}=\frac{2 d^{2}}{d+1}\left(I-\| v_{0}\right\rangle\right\rangle\left\langle\left\langle v_{0} \|\right) .\right.
\end{array}
$$

Proof: Some of this is a straightforward consequence of the fact that $J_{r}$ is the adjoint representative of $\Pi_{r}$. Since $\sum_{s} \Pi_{s}=d I$, we must have

$$
\sum_{s, t} J_{r s t} \Pi_{t}=\sum_{s} \operatorname{ad}_{\Pi_{r}} \Pi_{s}=0 .
$$

In view of the antisymmetry of the $J_{r s t}$ it follows that the sum over the $J_{r}$ is zero and hence $\left.\left.J_{r} \| v_{0}\right\rangle\right\rangle=0$. Using the relations

$$
Q_{r}=\frac{1}{2} J_{r}\left(J_{r}+I\right), \quad Q_{r}^{\mathrm{T}}=\frac{1}{2} J_{r}\left(J_{r}-I\right), \quad \bar{R}_{r}=J_{r}^{2},
$$


we deduce $\left.\left.\left.\left.\left.\left.Q_{r} \| v_{0}\right\rangle\right\rangle=Q_{r}^{\mathrm{T}} \| v_{0}\right\rangle\right\rangle=\bar{R}_{r} \| v_{0}\right\rangle\right\rangle=0$. It remains to prove Eqs. (166) and (167). It follows from Eq. (93) and some simple algebra that

$$
\sum_{r} Q_{r s t}=\frac{d+1}{d} \sum_{r} T_{r s t}-2 \sum_{r}\left\langle\left\langle s \| e_{r}\right\rangle\right\rangle\left\langle\left\langle e_{r} \| t\right\rangle\right\rangle=\frac{d^{2} \delta_{s t}-1}{d+1},
$$

from which it follows

$$
\left.\sum_{r} Q_{r}=\sum_{r} Q_{r}^{\mathrm{T}}=\frac{d^{2}}{d+1}\left(I-\| v_{0}\right\rangle\right\rangle\left\langle\left\langle v_{0} \|\right) .\right.
$$

Equation (167) follows from this and the fact that $R_{r}=Q_{r}+Q_{r}^{\mathrm{T}}$.

\section{GEOMETRICAL CONSIDERATIONS}

In this section we show that there are some interesting geometrical relationships between the subspaces onto which the operators $Q_{r}, Q_{r}^{\mathrm{T}}$, and $\bar{R}_{r}$ project. The original motivation for this work was an observation concerning the subspaces onto which the $\bar{R}_{r}$ project. $\bar{R}_{r}$ is a real matrix, and so it defines a $2(d-2)$ subspace in $\mathbb{R}^{d^{2}}$, which we will denote $\mathcal{R}_{r}$. We noticed that for each pair of distinct indices $r$ and $s$ the intersection $\mathcal{R}_{r} \cap \mathcal{R}_{s}$ is a one-dimensional line. This led us to speculate that a set of hyperplanes parallel to the $\mathcal{R}_{r}$ might be the edges of an interesting polytope. We continue to think that this could be the case, but we have unfortunately not been able to prove it. However, it appears to us that the results we obtained while trying to prove it might have an interest which is independent of the truth of the motivating speculation.

We will begin with some terminology. Let $P$ be any projector (on either $\mathbb{R}^{N}$ or $\mathbb{C}^{N}$ ), let $\mathcal{P}$ be the subspace onto which $P$ projects, and let $|\psi\rangle$ be any nonzero vector. Then we define the angle between $|\psi\rangle$ and $\mathcal{P}$ in the usual way, to be

$$
\theta=\cos ^{-1}\left(\frac{\| P|\psi\rangle \|}{\||\psi\rangle \|}\right)
$$

(so $\theta$ is the smallest angle between $|\psi\rangle$ and any of the vectors in $\mathcal{P}$ ).

Now suppose that $P^{\prime}$ is another projector and let $\mathcal{P}^{\prime}$ be the subspace onto which $P^{\prime}$ projects. We will say that $\mathcal{P}^{\prime}$ is uniformly inclined to $\mathcal{P}$ if every vector in $\mathcal{P}^{\prime}$ makes the same angle $\theta$ with $\mathcal{P}$. If $\theta=0$ this means that $\mathcal{P}^{\prime} \subseteq \mathcal{P}$, while if $\theta=\frac{\pi}{2}$ it means $\mathcal{P}^{\prime} \perp \mathcal{P}$. Suppose, on the other hand, that $0<\theta<\frac{\pi}{2}$. Let $\left|u_{1}^{\prime}\right\rangle, \ldots,\left|u_{n}^{\prime}\right\rangle$ be any orthonormal basis for $\mathcal{P}^{\prime}$, and define $\left|u_{r}\right\rangle=\sec \theta P\left|u_{r}^{\prime}\right\rangle$. Then $\left\langle u_{r} \mid u_{r}\right\rangle=1$ for all $r$. Moreover, if $P$ and $P^{\prime}$ are complex projectors,

$$
\left\langle u_{r}^{\prime}+e^{i \phi} u_{s}^{\prime}|P| u_{r}^{\prime}+e^{i \phi} u_{s}^{\prime}\right\rangle=2 \cos ^{2} \theta\left(1+\operatorname{Re}\left(e^{i \phi}\left\langle u_{r} \mid u_{s}\right\rangle\right)\right)
$$

for all $\phi$ and $r \neq s$. On the other hand it follows from the assumption that $\mathcal{P}^{\prime}$ is uniformly inclined to $\mathcal{P}$ that

$$
\left\langle u_{r}^{\prime}+e^{i \phi} u_{s}^{\prime}|P| u_{r}^{\prime}+e^{i \phi} u_{s}^{\prime}\right\rangle=2 \cos ^{2} \theta
$$

for all $\phi$ and $r \neq s$. It follows that $\left\langle u_{r} \mid u_{s}\right\rangle=\delta_{r s}$ for all $r, s$. It is easily seen that the same is true if $P$ and $P^{\prime}$ are real projectors.

Suppose we now make the further assumption that $\operatorname{dim}\left(\mathcal{P}^{\prime}\right)=\operatorname{dim}(\mathcal{P})=n$. Then $\left|u_{1}\right\rangle, \ldots,\left|u_{n}\right\rangle$ is an orthonormal basis for $\mathcal{P}$, and we have the completeness relation $P=\sum_{r}\left|u_{r}\right\rangle\left\langle u_{r}\right|$ and similar for $P^{\prime}$. From here it is easily shown that $\mathcal{P}$ is uniformly inclined to $\mathcal{P}^{\prime}$ with angle $\theta$.

It follows from the completeness relations for $P$ and $P^{\prime}$ that $P P^{\prime} P=\cos ^{2} \theta P$ and similar with $P$ and $P^{\prime}$ switched. Either one of these two conditions is not only necessary but also sufficient for the subspaces to be uniformly inclined. The proof is again straightforward linear algebra using the completeness relation in terms of the $\left|u_{r}\right\rangle$ and $\left|u_{r}^{\prime}\right\rangle$, so we omit the details.

It will be convenient to summarize all this in the form of a lemma. 
Lemma 15: Let $\mathcal{P}$ and $\mathcal{P}^{\prime}$ be any two subspaces, real or complex, having the same dimension $n$. Let $P$ and $P^{\prime}$ be the corresponding projectors. Then the following statements are equivalent.

(a) $\mathcal{P}$ is uniformly inclined to $\mathcal{P}^{\prime}$ at angle $\theta$.

(b) $P P^{\prime} P=\cos ^{2} \theta P$.

Suppose these conditions are satisfied for some $\theta$ in the range $0<\theta<\frac{\pi}{2}$, and let $\left|u_{1}\right\rangle, \ldots\left|u_{n}\right\rangle$ be any orthonormal basis for $\mathcal{P}$. Then there exists an orthonormal basis $\left|u_{1}^{\prime}\right\rangle, \ldots,\left|u_{n}^{\prime}\right\rangle$ for $\mathcal{P}^{\prime}$ such that $P^{\prime}\left|u_{r}\right\rangle=\cos \theta\left|u_{r}^{\prime}\right\rangle$ and $P\left|u_{r}^{\prime}\right\rangle=\cos \theta\left|u_{r}\right\rangle$.

Proof: Follows immediately from the above discussion.

We are now in a position to state the main results of this section. Let $\mathcal{Q}_{r}$ (respectively, $\overline{\mathcal{Q}}_{r}$ ) be the subspace onto which $Q_{r}$ (respectively, $Q_{r}^{\mathrm{T}}$ ) projects. We then have

Theorem 16: For each pair of distinct indices $r, s$ the subspace $\mathcal{Q}_{r}$ has the decomposition $\mathcal{Q}_{r}=\mathcal{Q}_{r s}^{0} \oplus \mathcal{Q}_{r s}$, where $\mathcal{Q}_{r s}^{0}$ and $\mathcal{Q}_{r s}$ are orthogonal and have dimensions $\operatorname{dim}\left(\mathcal{Q}_{r s}^{0}\right)=1$ and $\operatorname{dim}\left(\mathcal{Q}_{r s}\right)=d-2$, respectively. The relations between two such subspaces $\mathcal{Q}_{r}$ and $\mathcal{Q}_{s}$ are as follows.

(1) $\mathcal{Q}_{r s}^{0} \perp \mathcal{Q}_{s r}$ and $\mathcal{Q}_{r s} \perp \mathcal{Q}_{s r}^{0}$.

(2) $\mathcal{Q}_{r s}^{0}$ and $\mathcal{Q}_{s r}^{0}$ are inclined at angle $\cos ^{-1}\left(\frac{1}{d+1}\right)$.

(3) $\mathcal{Q}_{r s}$ and $\mathcal{Q}_{s r}$ are uniformly inclined at angle $\cos ^{-1}\left(\frac{1}{\sqrt{d+1}}\right)$.

All of the analogous statements from above also hold for $\overline{\mathcal{Q}}_{r}$. Furthermore, the relationship between the subspaces $\mathcal{Q}_{r}$ and $\overline{\mathcal{Q}}_{s}$ is as follows.

(1) $\mathcal{Q}_{r s}^{0} \perp \overline{\mathcal{Q}}_{s r}, \mathcal{Q}_{r s} \perp \overline{\mathcal{Q}}_{s r}^{0}$, and $\mathcal{Q}_{r s} \perp \overline{\mathcal{Q}}_{s r}$.

(2) $\mathcal{Q}_{r s}^{0}$ and $\overline{\mathcal{Q}}_{s r}^{0}$ are inclined at angle $\cos ^{-1}\left(\frac{d}{d+1}\right)$.

We will prove this theorem below. Before doing so, however, let us state the other main result of this section. Let $\mathcal{R}_{r}$ be the subspace onto which the $\bar{R}_{r}$ project. Since $\bar{R}_{r}$ is a real matrix we regard $\mathcal{R}_{r}$ as a subspace of $\mathbb{R}^{d^{2}}$. We have

Theorem 17: For each pair of distinct indices $r, s$ the subspace $\mathcal{R}_{r}$ has the decomposition

$$
\mathcal{R}_{r}=\mathcal{R}_{r s}^{0} \oplus \mathcal{R}_{r s}^{1} \oplus \mathcal{R}_{r s}
$$

where $\mathcal{R}_{r s}^{0}, \mathcal{R}_{r s}^{1}$, and $\mathcal{R}_{r s}$ are pairwise orthogonal and have the dimensions

$$
\operatorname{dim}\left(\mathcal{R}_{r s}^{0}\right)=1 \quad \operatorname{dim}\left(\mathcal{R}_{r s}^{1}\right)=1 \quad \operatorname{dim}\left(\mathcal{R}_{r s}\right)=2 d-4 .
$$

The relationships between these spaces are as follows.

1. $\mathcal{R}_{r s}^{0}=\mathcal{R}_{s r}^{0}$.

2. $\mathcal{R}_{r s}^{1} \perp \mathcal{R}_{s r}$ and $\mathcal{R}_{r s} \perp \mathcal{R}_{s r}^{1}$.

3. $\quad \mathcal{R}_{r s}^{1}$ and $\mathcal{R}_{s r}^{1}$ are inclined at angle $\cos ^{-1}\left(\frac{d-1}{d+1}\right)$.

4. $\mathcal{R}_{r s}$ and $\mathcal{R}_{s r}$ are uniformly inclined at angle $\cos ^{-1}\left(\sqrt{\frac{1}{d+1}}\right)$.

In particular, the subspaces $\overline{\mathcal{R}}_{r}$ and $\overline{\mathcal{R}}_{s}$ intersect in a line.

\section{A. Proof of Theorem 16}

Let $\left.\left.\| 1\rangle\rangle, \ldots, \| d^{2}\right\rangle\right\rangle$ be the standard basis for $\mathcal{H}_{d^{2}}$, as defined by Eq. (90). For each pair of distinct indices $r, s$ define

$$
\left.\left.\left.\left.\| f_{r s}\right\rangle\right\rangle=i \sqrt{d+1} Q_{r} \| s\right\rangle\right\rangle
$$


and let $\left.\left.\| f_{r s}^{*}\right\rangle\right\rangle$ be the conjugate vector. The significance of these vectors is that $\left.\left.\| f_{r s}\right\rangle\right\rangle\left\langle\left\langle f_{r s} \|\right.\right.$ (respectively, $\left.\| f_{r s}^{*}\right\rangle\left\langle\left\langle f_{r s}^{*} \|\right)\right.$ will turn out to be the projector onto the one-dimensional subspace $\mathcal{Q}_{r s}^{0}$ (respectively, $\overline{\mathcal{Q}}_{r s}^{0}$ ).

It is easily seen that $\left.\left.\| f_{r s}\right\rangle\right\rangle$ and $\left.\left.\| f_{r s}^{*}\right\rangle\right\rangle$ are normalized by using Eqs. (89) and (93). By using the fact that $Q_{r} Q_{r}^{\mathrm{T}}=0$, and of course since $Q_{r}$ is Hermitian $Q_{r}^{\mathrm{T}}=Q_{r}^{*}$, we also have $\left.\left\langle f_{r s} \| f_{r s}^{*}\right\rangle\right\rangle=0$ for all $r \neq s$. Note that, although we require that $r \neq s$ in the definitions of $\left.\left.\left.\| f_{r s}\right\rangle\right\rangle, \| f_{r s}^{*}\right\rangle$, the definitions continue to make sense when $r=s$. However, the vectors are then zero [as can be seen by setting $r=s$ in Eq. (94)].

The vectors $\left.\left.\left.\| f_{r s}\right\rangle, \| f_{r s}^{*}\right\rangle\right\rangle$ satisfy a number of additional identities, which it will be convenient to collect in a lemma.

Lemma 18: Let $\left.\| e_{r}\right\rangle$ be the (real) vector defined by Eq. (89). Then for all $r \neq s$ we have

$$
\left.\left.\left.\left.\left.\left.\left.\left.\| f_{r s}\right\rangle\right\rangle=-\| f_{s r}^{*}\right\rangle\right\rangle+i \sqrt{\frac{2}{d}}\left(\| e_{s}\right\rangle\right\rangle-\| e_{r}\right\rangle\right\rangle\right)
$$

Furthermore, we have

$$
\begin{gathered}
\left.\left.\left.\left.\left.Q_{r} \| f_{r s}\right\rangle\right\rangle=\| f_{r s}\right\rangle, \quad Q_{r}^{\mathrm{T}} \| f_{r s}\right\rangle\right\rangle=0, \\
\left.\left.\left.\left.\left.\left.Q_{s} \| f_{r s}\right\rangle\right\rangle=-\frac{1}{d+1} \| f_{s r}\right\rangle, \quad Q_{s}^{\mathrm{T}} \| f_{r s}\right\rangle\right\rangle=-\frac{d}{d+1} \| f_{s r}^{*}\right\rangle, \\
\left\langle\left\langle f_{r s} \| f_{s r}\right\rangle\right\rangle=\left\langle\left\langle f_{r s}^{*} \| f_{s r}^{*}\right\rangle\right\rangle=-\frac{1}{d+1} \\
\left\langle\left\langle f_{r s} \| f_{s r}^{*}\right\rangle\right\rangle=\left\langle\left\langle f_{r s}^{*} \| f_{s r}\right\rangle\right\rangle=-\frac{d}{d+1} .
\end{gathered}
$$

Proof: It follows from Eqs. (89) and (93) together with the relation $T_{r t s}=T_{s r t}$ that

$$
\left\langle\left\langle t \| f_{r s}\right\rangle\right\rangle+\left\langle\left\langle t \| f_{s r}^{*}\right\rangle\right\rangle=i \sqrt{\frac{2}{d}}\left(\left\langle\left\langle t \| e_{s}\right\rangle\right\rangle-\left\langle\left\langle t \| e_{r}\right\rangle\right\rangle\right),
$$

which then easily establishes Eq. (178).

Equation (179) is immediate consequences of the definitions and the fact that $Q_{r} Q_{r}^{\mathrm{T}}=0$. Turning to the proof of Eq. (180), it follows from Eqs. (92) and (93) that $\left.\left.Q_{s} \| e_{s}\right\rangle\right\rangle=0$. Using this and the fact that $\left.\left.Q_{s} \| f_{s r}^{*}\right\rangle\right)=0$ in Eq. (178), we find

$$
\left.\left.\left.\left.Q_{s} \| f_{r s}\right\rangle\right\rangle=-i \sqrt{\frac{2}{d}} Q_{s} \| e_{r}\right\rangle\right\rangle
$$

Since

$$
\left.\left.\left.\left.\left.\left.\| e_{r}\right\rangle\right\rangle=\sqrt{\frac{d}{2(d+1)}}(\| r\rangle\right\rangle+\| v_{0}\right\rangle\right\rangle\right)
$$

and taking account of the fact that $\left.\left.Q_{s} \| v_{0}\right\rangle\right\rangle=0$ [see Eq. (165)], we deduce

$$
\left.\left.\left.\left.\left.\left.Q_{s} \| f_{r s}\right\rangle\right\rangle=-i \sqrt{\frac{1}{d+1}} Q_{s} \| r\right\rangle\right\rangle=-\frac{1}{d+1} \| f_{s r}\right\rangle\right\rangle .
$$

The second identity in Eq. (180) follows in the same way by acting on both sides of Eq. (178) with $Q_{s}^{\mathrm{T}}$.

The last group of identities follows easily from considering quantities such as $\left.\left\langle f_{r s}\left\|Q_{r}\right\| f_{s r}\right\rangle\right\rangle$, together with the above expressions and taking some complex conjugates. 
This lemma provides a substantial part of what we need to prove the theorem. The remaining part is provided by

Lemma 19: For all $r \neq s$

$$
\begin{aligned}
& \left.\left.Q_{r} Q_{s} Q_{r}=\frac{1}{d+1} Q_{r}-\frac{d}{(d+1)^{2}} \| f_{r s}\right\rangle\right\rangle\left\langle f_{r s} \|,\right. \\
& \left.\left.Q_{r} Q_{s}^{\mathrm{T}} Q_{r}=\frac{d^{2}}{(d+1)^{2}} \| f_{r s}\right\rangle\right\rangle\left\langle f_{r s} \| .\right.
\end{aligned}
$$

Proof: We will only prove the first identity. The second identity has a similar proof. It follows from Eq. (93) that

$$
\left.\left.Q_{r} Q_{s} Q_{r}=\frac{d+1}{d} Q_{r} T_{s} Q_{r}-2 Q_{r} \| e_{s}\right\rangle\right\rangle\left\langle\left\langle e_{s} \| Q_{r} .\right.\right.
$$

In view of Eqs. (185) and (165), and the definition of $\left.\| f_{r s}\right\rangle$, we have

$$
\left.\left.\left.\left.\left.\left.Q_{r} \| e_{s}\right\rangle\right\rangle=\sqrt{\frac{d}{2(d+1)}} Q_{r} \| s\right\rangle\right\rangle=-i \frac{\sqrt{d}}{\sqrt{2}(d+1)} \| f_{r s}\right\rangle\right\rangle .
$$

Substituting this expression into Eq. (189), we obtain

$$
\left.\left.Q_{r} Q_{s} Q_{r}=\frac{d+1}{d} Q_{r} T_{s} Q_{r}-\frac{d}{(d+1)^{2}} \| f_{r s}\right\rangle\right\rangle\left\langle f_{r s} \| .\right.
$$

The problem therefore reduces to showing

$$
Q_{r} T_{s} Q_{r}=\frac{d}{(d+1)^{2}} Q_{r} .
$$

Using Eq. (93) we find

$$
\begin{aligned}
\left\langle\left\langle a\left\|Q_{r} T_{s} Q_{r}\right\| b\right\rangle\right\rangle= & \frac{(d+1)^{2}}{d^{2}}\left\langle\left\langle a\left\|T_{r} T_{s} T_{r}\right\| b\right\rangle\right\rangle+\frac{2(d+1)}{d} K_{r a}^{2} K_{r b}^{2}\left\langle\left\langle e_{r}\left\|T_{s}\right\| e_{r}\right\rangle\right\rangle \\
& -\frac{1}{2}\left(\frac{2(d+1)}{d}\right)^{\frac{3}{2}}\left(K_{r a}^{2}\left\langle\left\langle e_{r}\left\|T_{s} T_{r}\right\| b\right\rangle\right\rangle+K_{r b}^{2}\left\langle\left\langle a\left\|T_{r} T_{s}\right\| e_{r}\right\rangle\right\rangle\right) .
\end{aligned}
$$

Using the definitions of $\left.T_{r}, \| e_{r}\right\rangle$ and Eq. (23) (the 2-design property) we find, after some algebra,

$$
\begin{aligned}
& \left\langle\left\langle a\left\|T_{r} T_{s} T_{r}\right\| b\right\rangle=\frac{d^{2}}{(d+1)^{2}}\left(K_{r a}^{2} T_{r s b}+K_{r b}^{2} T_{r a s}+K_{r s}^{2} T_{r a b}+K_{r a}^{2} K_{r b}^{2}\right),\right. \\
& \left\langle\left\langle e_{r}\left\|T_{s} T_{r}\right\| b\right\rangle\right\rangle=2\left(\frac{d}{2(d+1)}\right)^{\frac{3}{2}}\left(2 K_{r s}^{2} K_{r b}^{2}+K_{r b}^{2}+T_{r s b}\right), \\
& \left.\left\langle a\left\|T_{r} T_{s}\right\| e_{r}\right\rangle\right\rangle=2\left(\frac{d}{2(d+1)}\right)^{\frac{3}{2}}\left(2 K_{r s}^{2} K_{r a}^{2}+K_{r a}^{2}+T_{r a s}\right), \\
& \left\langle\left\langle e_{r}\left\|T_{s}\right\| e_{r}\right\rangle\right\rangle=\frac{d}{2(d+1)}\left(3 K_{r s}^{2}+1\right) .
\end{aligned}
$$

Substituting these expressions into Eq. (193), we deduce Eq. (192).

The proof of the second identity is essentially identical, except that it also uses the fact that $G_{r a} G_{a s} G_{s b} G_{b r}=(d+1) T_{r a s} T_{r s b}$ (in view of the fact that $r \neq s$ ). 
Now define the rank $d-1$ projectors

$$
\left.Q_{r s}=Q_{r}-\| f_{r s}\right\rangle\left\langle\left\langle f_{r s}\left\|, \quad Q_{r s}^{\mathrm{T}}=Q_{r}^{\mathrm{T}}-\right\| f_{r s}^{*}\right\rangle\right\rangle\left\langle f_{r s}^{*} \|,\right.
$$

and let $\mathcal{Q}_{r s}^{0}, \mathcal{Q}_{r s}, \overline{\mathcal{Q}}_{r s}^{0}$, and $\overline{\mathcal{Q}}_{r s}$ be, respectively, the subspaces onto which $\left.\left.\| f_{r s}\right\rangle\right\rangle\left\langle f_{r s} \|, Q_{r s}\right.$, $\left.\left.\| f_{r s}^{*}\right\rangle\right\rangle\left\langle\left\langle f_{r s}^{*} \|\right.\right.$, and $Q_{r s}^{*}$ project. It is immediate that we have the orthogonal decompositions

$$
\mathcal{Q}_{r}=\mathcal{Q}_{r s}^{0} \oplus \mathcal{Q}_{r s}, \quad \overline{\mathcal{Q}}_{r}=\overline{\mathcal{Q}}_{r s}^{0} \oplus \overline{\mathcal{Q}}_{r s} .
$$

Using Lemma 18 we find

$$
\left.\left.\left.\left.Q_{s r} \| f_{r s}\right\rangle\right\rangle=Q_{r s} \| f_{s r}\right\rangle\right\rangle=0
$$

implying that $\mathcal{Q}_{r s}^{0} \perp \mathcal{Q}_{s r}$ and $\mathcal{Q}_{r s} \perp \mathcal{Q}_{s r}^{0}$, and

$$
\left|\left\langle f_{r s} \| f_{s r}\right\rangle\right\rangle \mid=\frac{1}{d+1},
$$

which means that $\mathcal{Q}_{r s}^{0}$ and $\mathcal{Q}_{s r}^{0}$ are inclined at angle $\cos ^{-1}\left(\frac{1}{d+1}\right)$. Using Lemma 18 together with Lemma 19 we find

$$
\left.\left.\left.Q_{r s} Q_{s r} Q_{r s}=Q_{r} Q_{s} Q_{r}-\| f_{r s}\right\rangle\right\rangle\left\langle f_{r s}\left\|Q_{s} Q_{r}-Q_{r} Q_{s}\right\| f_{r s}\right\rangle\right\rangle\left\langle f_{r s} \|=\frac{1}{d+1} Q_{r s},\right.
$$

which in view of Lemma 15 implies that $\mathcal{Q}_{r s}$ and $\mathcal{Q}_{s r}$ are uniformly inclined at angle $\cos ^{-1}\left(\frac{1}{\sqrt{d+1}}\right)$. This proves the first part of the theorem; the related statements are proved similarly. This completes the proof of Theorem 16 .

\section{B. Proof of Theorem 17}

Define

$$
\left.\left.\left.\left.\left.\left.\left.\left.\left.\left.\| g_{r s}\right\rangle\right\rangle=\frac{1}{\sqrt{2}}\left(\| f_{r s}^{*}\right\rangle\right\rangle+\| f_{r s}\right\rangle\right), \quad \| \bar{g}_{r s}\right\rangle\right\rangle=\frac{i}{\sqrt{2}}\left(\| f_{r s}^{*}\right\rangle\right\rangle-\| f_{r s}\right\rangle\right) .
$$

By construction the components of $\left.\left.\| g_{r s}\right\rangle\right\rangle$ and $\left.\| \bar{g}_{r s}\right\rangle$ in the standard basis are real, so we can regard them as $\in \mathbb{R}^{d^{2}}$. Clearly they are orthonormal. It is also readily verified, using Lemma 18 , that they are +1 eigenvectors of $\bar{R}_{r}$. Therefore,

$$
\left.\left.R_{r s}=\bar{R}_{r}-\| g_{r s}\right\rangle\right\rangle\left\langle\left\langle g_{r s}\|-\| \bar{g}_{r s}\right\rangle\right\rangle\left\langle\bar{g}_{r s} \|\right.
$$

is a rank $2 d-4$ projector. If we define $\mathcal{R}_{r s}^{0}, \mathcal{R}_{r s}^{1}$, and $\mathcal{R}_{r s}$ to be, respectively, the subspaces onto which $\left.\left.\| g_{r s}\right\rangle\right\rangle\left\langle\left\langle g_{r s}\|,\| \bar{g}_{r s}\right\rangle\right\rangle\left\langle\bar{g}_{r s} \|\right.$, and $R_{r s}$ project we have the orthogonal decomposition

$$
\mathcal{R}_{r}=\mathcal{R}_{r s}^{0} \oplus \mathcal{R}_{r s}^{1} \oplus \mathcal{R}_{r s} .
$$

It follows from Eq. (178) and its conjugate equation that $\left.\left.\left.\| g_{r s}\right\rangle\right\rangle=-\| g_{s r}\right\rangle$, which implies that $\mathcal{R}_{r s}^{0}=\mathcal{R}_{s r}^{0}$ for all $r \neq s$. It is also easily verified, using Lemma 18 , that

$$
\left|\left\langle\bar{g}_{r s} \| \bar{g}_{s r}\right\rangle\right\rangle \mid=\frac{d-1}{d+1}
$$

from which it follows that $\mathcal{R}_{r s}^{1}$ and $\mathcal{R}_{s r}^{1}$ are inclined at angle $\cos ^{-1}\left(\frac{d-1}{d+1}\right)$. We next observe that

$$
R_{r s}=Q_{r s}+Q_{r s}^{\mathrm{T}} .
$$

Using Lemma 18 once again we deduce $\left.\left.R_{r s} \| \bar{g}_{s r}\right\rangle=R_{s r} \| \bar{g}_{r s}\right\rangle=0$, which implies that $\mathcal{R}_{r s}^{1} \perp \mathcal{R}_{s r}$ and $\mathcal{R}_{r s} \perp \mathcal{R}_{s r}^{1}$. Finally, we know from Theorem 16 that $Q_{r s}^{\mathrm{T}} Q_{s r}=Q_{r s} Q_{s r}^{\mathrm{T}}=0$, hence

$$
R_{r s} R_{s r} R_{r s}=Q_{r s} Q_{s r} Q_{r s}+Q_{r s}^{\mathrm{T}} Q_{s r}^{\mathrm{T}} Q_{r s}^{\mathrm{T}}=\frac{1}{d+1} R_{r s} .
$$

In view of Lemma 15 it follows that $\mathcal{R}_{r s}$ and $\mathcal{R}_{s r}$ are uniformly inclined at angle $\cos ^{-1}\left(\frac{1}{\sqrt{d+1}}\right)$. 


\section{Further identities}

We conclude this section with another set of identities involving the vectors $\left.\left.\left.\left.\left.\left.\| f_{r s}\right\rangle\right\rangle, \| f_{r s}^{*}\right\rangle\right\rangle, \| g_{r s}\right\rangle\right\rangle$, and $\left.\| \bar{g}_{r s}\right\rangle$.

Define

$$
\left.\left.\left.\left.\left.\left.\| \bar{e}_{r}\right\rangle\right\rangle=\sqrt{\frac{2 d}{d-1}} \| e_{r}\right\rangle\right\rangle-\sqrt{\frac{d+1}{d-1}} \| v_{0}\right\rangle\right\rangle
$$

where $\left.\left.\| v_{0}\right\rangle\right\rangle$ is the vector defined by Eq. (164). It is readily verified that $\left\langle\left\langle\bar{e}_{r} \| \bar{e}_{r}\right\rangle\right\rangle=1$ and $\left\langle\left\langle\bar{e}_{r} \| v_{0}\right\rangle\right\rangle=0$, so $\left.\left.\| \bar{e}_{r}\right\rangle\right\rangle$ and $\left.\left.\| v_{0}\right\rangle\right\rangle$ are orthonormal basis for the two-dimensional subspace spanned by $\left.\left.\left.\left.\| e_{r}\right\rangle\right\rangle, \| v_{0}\right\rangle\right\rangle$. Note that

$$
\left.\left.\left.\left.\left.\left.Q_{r} \| \bar{e}_{r}\right\rangle\right\rangle=Q_{r}^{\mathrm{T}} \| \bar{e}_{r}\right\rangle\right\rangle=\bar{R}_{r} \| \bar{e}_{r}\right\rangle\right\rangle=0
$$

We then have

Theorem 20: For all $r$

$$
\begin{gathered}
\left.\left.Q_{r}=\frac{1}{d+1} \sum_{s \neq r} \| f_{r s}\right\rangle\right\rangle\left\langle\left\langle f_{r s} \|,\right.\right. \\
\left.\left.\left.\bar{R}_{r}=\frac{2}{d+1} \sum_{s \neq r} \| \bar{g}_{r s}\right\rangle\right\rangle\left\langle\bar{g}_{r s}\left\|=\frac{2}{d+1} \sum_{s \neq r}\right\| g_{r s}\right\rangle\right\rangle\left\langle g_{r s} \|,\right. \\
\left.\left.\left.\frac{1}{d-1} \sum_{s \neq r} \| f_{s r}\right\rangle\right\rangle\left\langle f_{s r}\left\|=Q_{r}^{\mathrm{T}}+\right\| \bar{e}_{r}\right\rangle\right\rangle\left\langle\langle \overline { e } _ { r } \| + \frac { 1 } { d ^ { 2 } - 1 } ( I - \| v _ { 0 } \rangle \rangle \left\langle\left\langle v_{0} \|\right),\right.\right. \\
\left.\left.\frac{2}{d+1} \sum_{s \neq r} \| g_{s r}\right\rangle\right\rangle\left\langle g_{s r} \|=\bar{R}_{r},\right. \\
\left.\left.\left.\frac{2}{d-3} \sum_{s \neq r} \| \bar{g}_{s r}\right\rangle\right\rangle\left\langle\bar{g}_{s r}\left\|=\bar{R}_{r}+\frac{4(d-1)}{d-3}\right\| \bar{e}_{r}\right\rangle\right\rangle\left\langle\langle \overline { e } _ { r } \| + \frac { 4 } { ( d + 1 ) ( d - 3 ) } ( I - \| v _ { 0 } \rangle \rangle \left\langle\left\langle v_{0} \|\right) .\right.\right.
\end{gathered}
$$

Proof: The first identity follows from the definition of $\left.\| f_{r s}\right\rangle$ and the fact that $\left.\left.Q_{r} \| r\right\rangle\right\rangle=0$ [as can be seen by setting $r=s$ in Eq. (94)]. By similar reasoning, we also have

$$
\left.\left.\frac{1}{d+1} \sum_{s \neq r} \| f_{r s}\right\rangle\right\rangle\left\langle f_{r s}^{*} \|=-Q_{r} Q_{r}^{\mathrm{T}}=0 .\right.
$$

A similar relation holds by taking the complex conjugate on both sides. By manipulating the expressions relating $\left.\| g_{r s}\right\rangle$ in terms of $\left.\left.\| f_{r s}\right\rangle\right\rangle$ (and similar relations),

$$
\left.\left.\frac{2}{d+1} \sum_{s \neq r} \| g_{r s}\right\rangle\right\rangle\left\langle\left\langle g_{r s} \|=\bar{R}_{r} .\right.\right.
$$

The middle expression in Eq. (212) is proved similarly.

To prove the second group of identities we have to work a little harder. Using Eqs. (89) and (93) we find

$$
\sum_{s \neq r}\left\langle\left\langle a \| f_{s r}\right\rangle\right\rangle\left\langle\left\langle f_{s r} \| b\right\rangle\right\rangle=\frac{(d+1)^{3}}{d^{2}} \sum_{s}\left(T_{s a r} T_{s r b}-K_{s a}^{2} K_{s r}^{2} T_{s r b}-K_{s r}^{2} K_{s b}^{2} T_{s a r}+K_{s a}^{2} K_{s r}^{4} K_{s b}^{2}\right) .
$$

After some algebra we find

$$
\left.\sum_{s} T_{s a r} T_{s r b}=\frac{d}{d+1}\left[\left(\sqrt{\frac{d-1}{d+1}}\left\langle\left\langle a \| \bar{e}_{r}\right\rangle\right\rangle+\frac{1}{d}\right)\left(\sqrt{\frac{d-1}{d+1}}\left\langle\bar{e}_{r} \| b\right\rangle\right\rangle+\frac{1}{d}\right)+T_{r b a}\right],
$$




$$
\begin{aligned}
& \left.\sum_{s} K_{s a}^{2} K_{s r}^{2} T_{s r b}=\frac{d}{d+1}\left[\left(\sqrt{\frac{d-1}{d+1}}\left\langle\left\langle a \| \bar{e}_{r}\right\rangle\right\rangle+\frac{2 d+1}{d(d+1)}\right)\left(\sqrt{\frac{d-1}{d+1}}\left\langle\bar{e}_{r} \| b\right\rangle\right\rangle+\frac{1}{d}\right)+\frac{1}{d+1} T_{r b a}\right], \\
& \sum_{s} K_{s r}^{2} K_{s b}^{2} T_{s a r}=\frac{d}{d+1}\left[\left(\sqrt{\frac{d-1}{d+1}}\left\langle\left\langle a \| \bar{e}_{r}\right\rangle\right\rangle+\frac{1}{d}\right)\left(\sqrt{\frac{d-1}{d+1}}\left\langle\left\langle\bar{e}_{r} \| b\right\rangle\right\rangle+\frac{2 d+1}{d(d+1)}\right)+\frac{1}{d+1} T_{r b a}\right], \\
& \sum_{s} K_{s a}^{2} K_{s r}^{4} K_{s b}^{2}=\frac{d}{(d+1)}\left[\frac{d+2}{d+1}\left(\sqrt{\frac{d-1}{d+1}}\left\langle\left\langle a \| \bar{e}_{r}\right\rangle\right\rangle+\frac{1}{d}\right)\left(\sqrt{\frac{d-1}{d+1}}\left\langle\left\langle\bar{e}_{r} \| b\right\rangle\right\rangle+\frac{1}{d}\right)+\frac{d \delta_{a b}}{(d+1)^{3}}+\frac{d+2}{(d+1)^{3}}\right],
\end{aligned}
$$

where we used Eq. (23) to derive the first expression. Substituting these expressions into Eq. (218) and using

$$
\left\langle\left\langle a\left\|Q_{r}^{\mathrm{T}}\right\| b\right\rangle\right\rangle=\frac{d+1}{d}\left(T_{r b a}-\left(\sqrt{\frac{d-1}{d+1}}\left\langle\left\langle a \| \bar{e}_{r}\right\rangle\right\rangle+\frac{1}{d}\right)\left(\sqrt{\frac{d-1}{d+1}}\left\langle\left\langle\bar{e}_{r} \| b\right\rangle\right\rangle+\frac{1}{d}\right)\right),
$$

we deduce Eq. (213).

Equation (214) is an immediate consequence of Eq. (212) and the fact that $\left.\left.\left.\| g_{s r}\right\rangle=-\| g_{r s}\right\rangle\right\rangle$.

To prove Eq. (215) observe that it follows from Eqs. (213) and (214) that

$$
\left.\left.\frac{1}{2} \sum_{s \neq r}\left(\| f_{s r}\right\rangle\right\rangle\left\langle\left\langle f_{s r}^{*}\|+\| f_{s r}^{*}\right\rangle\right\rangle\left\langle\left\langle f_{s r} \|\right)=\bar{R}_{r}-(d-1) \| \bar{e}_{r}\right\rangle\right\rangle\left\langle\langle \overline { e } _ { r } \| - \frac { 1 } { d + 1 } ( I - \| v _ { 0 } \rangle \rangle \left\langle\left\langle v_{0} \|\right)\right.\right. \text {. }
$$

Hence

$$
\left.\left.\frac{2}{d-3} \sum_{s \neq r} \| \bar{g}_{s r}\right\rangle\right\rangle\left\langle\left\langle\bar{g}_{s r}\left\|=\bar{R}_{r}+\frac{4(d-1)}{d-3}\right\| \bar{e}_{r}\right\rangle\right\rangle\left\langle\langle \overline { e } _ { r } \| + \frac { 4 } { ( d + 1 ) ( d - 3 ) } ( I - \| v _ { 0 } \rangle \rangle \left\langle\left\langle v_{0} \|\right) .\right.\right.
$$

\section{THE $\boldsymbol{P}-\boldsymbol{P}^{\mathrm{T}}$ PROPERTY}

In Secs. II-IX the $Q-Q^{\mathrm{T}}$ property has played a prominent role. In this section we show that in the particular case of a Weyl-Heisenberg covariant SIC-POVM, and with the appropriate choice of gauge, the Gram projector [defined in Eq. (48)] has an analogous property, which we call the $P-P^{T}$ property. Specifically, one has $\left.P P^{\mathrm{T}}=P^{\mathrm{T}} P=\| h\right\rangle\langle\langle h \|$, where $\| h\rangle\rangle$ is a normalized vector whose components in the standard basis are all real. In odd dimensions the components of $\| h\rangle$ in the standard basis can be simply expressed in terms of the Wigner function of the fiducial vector. It could be said that the projectors $P$ and $P^{\mathrm{T}}$ are almost orthogonal (by contrast with the projectors $Q_{r}$ and $Q_{r}^{\mathrm{T}}$ which are completely orthogonal). More precisely, $P$ has the spectral decomposition $P=\bar{P}+\| h\rangle\rangle\left\langle h \|\right.$, where $\bar{P}$ is a rank $(d-1)$ projector with the property $\bar{P} \bar{P}^{\mathrm{T}}=0$. This means that the matrix $J_{P}=P-P^{\mathrm{T}}$ is a pure imaginary Hermitian matrix with the property that $J_{P}^{2}$ is a real rank $2 d-2$ projector (c.f. the discussion in Sec. V).

Although we are mainly interested in the $P-P^{\mathrm{T}}$ property as it applies to SIC-POVMs, it should be noted that it actually holds for any Weyl-Heisenberg covariant POVM (with the appropriate choice of gauge), which we now consider.

Let us begin by fixing notation. Let $|0\rangle, \ldots,|d-1\rangle$ be an orthonormal basis for $d$-dimensional Hilbert space and let $X$ and $Z$ be the operators whose action on the $|r\rangle$ is

$$
X|a\rangle=|a+1\rangle, \quad Z|a\rangle=\omega^{a}|a\rangle,
$$

where $\omega=e^{\frac{2 \pi i}{d}}$ and the addition of indices in the first equation is $\bmod d$. We then define the Weyl-Heisenberg displacement operators by (adopting the convention used in, for example, Ref. 16)

$$
D_{\mathbf{p}}=\tau^{p_{1} p_{2}} X^{p_{1}} Z^{p_{2}}
$$


where $\mathbf{p}$ is the vector $\left(p_{1}, p_{2}\right)\left(p_{1}, p_{2}\right.$ being integers $)$ and $\tau=e^{\frac{(d+1) \pi i}{d}}$. Generally speaking the decision to insert the phase $\tau^{p_{1} p_{2}}$ is a matter of convention, and many authors define it differently or else omit altogether. However, for the purposes of this section it is essential, as a different choice of phase at this stage would lead to a different gauge in the class of POVMs to be defined below, and the Gram projector would then typically not have the $P-P^{\mathrm{T}}$ property.

Note that $\tau^{2}=\tau^{d^{2}}=\omega$ in every dimension. If the dimension is odd we can write $\tau=\omega^{\frac{d+1}{2}}$. So $\tau$ is a $d$ th root of unity. However, if the dimension is even, $\tau^{d}=-1$. This has the consequence that

$$
D_{\mathbf{p}+d \mathbf{u}}=(-1)^{u_{1} p_{2}+u_{2} p_{1}} D_{\mathbf{p}}
$$

So in even dimension $\mathbf{p}=\mathbf{q}(\bmod d)$ does not necessarily imply $D_{\mathbf{p}}=D_{\mathbf{q}}$ [although the operators are, of course, equal if $\mathbf{p}=\mathbf{q}(\bmod 2 d)]$

In every dimension (even or odd) we have the following properties for all $\mathbf{p}, \mathbf{q}$, and $n$ :

$$
D_{\mathbf{p}}^{\dagger}=D_{-\mathbf{p}}, \quad\left(D_{\mathbf{p}}\right)^{n}=D_{n \mathbf{p}}, \quad D_{\mathbf{p}} D_{\mathbf{q}}=\tau^{\langle\mathbf{p}, \mathbf{q}\rangle} D_{\mathbf{p}+\mathbf{q}} .
$$

In the last expression $\langle\mathbf{p}, \mathbf{q}\rangle$ is the symplectic form $\langle\mathbf{p}, \mathbf{q}\rangle=p_{2} q_{1}-p_{1} q_{2}$.

Now let $|\psi\rangle$ be any normalized vector (not necessarily a SIC-fiducial vector) and define $\left|\psi_{\mathbf{p}}\right\rangle=$ $D_{\mathbf{p}}|\psi\rangle$. Let

$$
L=\sum_{\mathbf{p} \in \mathbb{Z}_{d}^{2}}\left|\psi_{\mathbf{p}}\right\rangle\left\langle\psi_{\mathbf{p}}\right|
$$

It is easily seen that $\left[D_{\mathbf{p}}, L\right]=0$ for all $\mathbf{p}$.

We now appeal to the fact that there is no nontrivial subspace of $\mathcal{H}_{d}$ which the displacement operators leave invariant. To see this assume the contrary. Then there would exist nonzero vectors $|\phi\rangle,|\chi\rangle$ such that $\left\langle\phi\left|D_{\mathbf{p}}\right| \chi\right\rangle=0$ for all $\mathbf{p}$. Writing the left-hand side out in full this gives

$$
\sum_{a=0}^{d-1} \omega^{p_{2} a}\left\langle\phi \mid a+p_{1}\right\rangle\langle a \mid \chi\rangle=0
$$

for all $p_{1}, p_{2}$. Taking the discrete Fourier transform with respect to $p_{2}$, we have $\left\langle\phi \mid a+p_{1}\right\rangle\langle a \mid \chi\rangle=0$ for all $a, p_{1}$, implying that either $|\phi\rangle=0$ or $|\chi\rangle=0$ - contrary to assumption. We can therefore use Schur's lemma ${ }^{55}$ to deduce that $L=k I$ for some constant $k$. Taking the trace on both sides of this equation we infer that $k=d$. We conclude that $\frac{1}{d}\left|\psi_{\mathbf{p}}\right\rangle\left\langle\psi_{\mathbf{p}}\right|$ is a POVM. We refer to POVMs of this general class as Weyl-Heisenberg covariant POVMs. We refer to the vector $|\psi\rangle$ which generates the POVM as the fiducial vector (with no implication that it is necessarily a SIC-fiducial).

Now consider the Gram projector

$$
\left.\left.P=\sum_{\mathbf{p}, \mathbf{q} \in \mathbb{Z}_{d}^{2}} P_{\mathbf{p}, \mathbf{q}} \| \mathbf{p}\right\rangle\right\rangle\langle\mathbf{q} \|,
$$

where $P_{\mathbf{p}, \mathbf{q}}=\frac{1}{d}\left\langle\psi_{\mathbf{p}} \mid \psi_{\mathbf{q}}\right\rangle$ and where we label the matrix elements of $P$ and the standard basis kets with the vectors $\mathbf{p}, \mathbf{q}$, rather than with the single integer indices $r, s$ as in the rest of this paper. We know from Theorem 1 that $P$ is a rank- $d$ projector.

Using Eq. (229) we have

$$
\left\langle\langle\mathbf{p}\|P\| \mathbf{q}\rangle=\frac{1}{d} \sum_{a=0}^{d-1} \tau^{p_{1} p_{2}+q_{1} q_{2}} \omega^{a q_{2}-\left(q_{1}+a\right) p_{2}}\left\langle\psi \mid a+q_{1}-p_{1}\right\rangle\langle a \mid \psi\rangle .\right.
$$

Some further algebra shows that

$$
\left\langle\left\langle\mathbf{p}\left\|P P^{\mathrm{T}}\right\| \mathbf{q}\right\rangle=\langle\langle\mathbf{p} \| h\rangle\rangle\langle h \| \mathbf{q}\rangle\right\rangle
$$

where $\| h\rangle\rangle$ is the vector with components

$$
\langle\mathbf{p} \| h\rangle=\frac{1}{\sqrt{d}} \sum_{a=0}^{d-1} \tau^{p_{1} p_{2}} \omega^{p_{2} a}\left\langle\psi \mid-a-p_{1}\right\rangle\langle a \mid \psi\rangle .
$$


It is easily verified that $\| h\rangle\rangle$ is normalized and that $\langle\mathbf{p} \| h\rangle\rangle$ is real.

Finally, suppose that the dimension is odd. Then the Wigner function of the state $|\psi\rangle$ is ${ }^{56,57}$

$$
W(\mathbf{p})=\frac{1}{d}\left\langle\psi\left|D_{\mathbf{p}} U_{\mathrm{P}} D_{\mathbf{p}}^{\dagger}\right| \psi\right\rangle=\frac{1}{d}\left\langle\psi\left|D_{2 \mathbf{p}} U_{\mathrm{P}}\right| \psi\right\rangle,
$$

where $U_{\mathrm{P}}$ is the parity operator, whose action on the standard basis is $U_{\mathrm{P}}|a\rangle=|-a\rangle$. It is straightforward to show

$$
\langle\langle\mathbf{p} \| h\rangle\rangle=\sqrt{d} W\left(-2^{-1} \mathbf{p}\right),
$$

where $2^{-1}=(d+1) / 2$ is the multiplicative inverse of 2 considered as an element of $\mathbb{Z}_{d}$ : i.e., the unique integer $0 \leq m<d$ such that $2 m=1(\bmod d)$.

\section{CONCLUSION}

A curious fact about SIC-POVMs is that, although they are characterized by their being highly symmetric structures, they do not wear this property on their sleeve (so to speak). If one casually inspects the components of a SIC-fiducial, without knowing in advance that that is what they are, there does not seem to be anything special about them at all. Indeed, so far from there being any obvious pattern to the components, they seem, to a casual inspection, like a completely random collection of numbers. Moreover, this is just as true of an exact fiducial as it is of a numerical one (see, for instance, the tabulations in Scott and Grassl ${ }^{46}$ ). It is only when one looks at them through the right pair of spectacles, and takes the trouble to calculate the overlaps $\operatorname{Tr}\left(\Pi_{r} \Pi_{s}\right)$, that the symmetry becomes apparent. The situation is a little reminiscent of a hologram, which only takes on the aspect of a meaningful image when it is viewed in the right way. If one wanted to summarize the content of this paper in a nutshell it could be said that we have exhibited some other pairs of spectacles-other ways of looking at a SIC — which cause its inner secrets (or at any rate some of its inner secrets) to become manifest.

Rather than focusing on the SIC-vectors $\left|\psi_{r}\right\rangle$, as is usually done, we have focused on the angle tensors $\theta_{r s}$ and $\theta_{r s t}$ and on the $T, J$, and $R$ matrices defined in terms of them. This is an important change of emphasis because, rather than being tied to any particular SIC, these quantities characterize entire families of unitarily equivalent SICs. Like the components of a SIC-fiducial, the angle tensors appear, to a casual inspection, like a random collection of numbers. However, if one examines the spectra of the $T, J$, and $R$ matrices one realizes that, underlying the appearance of randomness, there is a high degree of order. If one then goes on to examine the geometrical relationships between the subspaces onto which the $Q, Q^{\mathrm{T}}$, and $\bar{R}$ matrices project, as we did in Sec. IX, one finds yet more instances of structure and order. To our minds what is particularly interesting about all of this is that none of it is obviously suggested by the defining property of a SIC, that $\operatorname{Tr}\left(\Pi_{r} \Pi_{s}\right)=1 /(d+1)$ for $r \neq s$.

In the course of this paper we have several times expressed the hope that the Lie algebraic perspective on a SIC will lead to a solution to the existence problem. Of course that is only a hope, and it may not be fulfilled. However, we feel on rather safer ground when we suggest that the solution is likely to come, if not from this investigation, then from one which is like it to the extent that it focuses on a feature of a SIC which is not immediately apparent.

Specializing to the case of a Weyl-Heisenberg covariant SIC, a fiducial vector $|\psi\rangle$ is a solution to the equations

$$
\left|\left\langle\psi\left|D_{\mathbf{p}}\right| \psi\right\rangle\right|^{2}=\frac{d \delta_{\mathbf{p}, \mathbf{0}}+1}{d+1} .
$$

Allowing for the arbitrariness of the overall phase of $|\psi\rangle$ and taking $|\psi\rangle$ to be normalized, this gives us $d^{2}-1$ conditions on only $2 d-2$ independent real parameters. The equations are thus overdetermined and very highly overdetermined when $d$ is large. Nevertheless, they have turned out to be soluble in every case which has been investigated to date. It seems likely that progress will depend on finding the structural feature which is responsible for this remarkable fact. The motivation for this paper is the belief that it may be structural features of the Lie algebra $\operatorname{gl}(d, \mathbb{C})$ which are 
responsible. That suggestion may or may not be correct. But if it turns out to be incorrect, the amount of effort which has been expended on this problem over a period of more than ten years, so far without fruit, suggests to us that the solution will depend on finding some other structural feature of a SIC, which is not obvious and which has hitherto escaped attention.

\section{ACKNOWLEDGMENTS}

The authors thank I. Bengtsson for discussions. The work of D.M.A. and C.A.F. was supported in part by the U. S. Office of Naval Research (Grant No. N00014-09-1-0247). Research at Perimeter Institute is supported by the Government of Canada through Industry Canada and by the Province of Ontario through the Ministry of Research and Innovation.

${ }^{1}$ S. G. Hoggar, Geom. Dedic. 69, 287 (1998).

${ }^{2}$ G. Zauner, "Quantum designs-Foundations of a non-commutative theory of designs" (in German), Ph.D. dissertation (University of Vienna, 1999). Available online at http://www.mat.univie.ac.at/neum/papers/physpapers.html.

${ }^{3}$ C. M. Caves, "Symmetric informationally complete POVMs," UNM Information Physics Group Internal Report, 1999. Available online at http://info.phys.unm.edu/ caves/reports/reports.html.

${ }^{4}$ C. A. Fuchs and M. Sasaki, Quantum Inf. Comput. 3, 277 (2003).

${ }^{5}$ J. M. Renes, R. Blume-Kohout, A. J. Scott, and C. M. Caves, J. Math. Phys. 45, 2171 (2004).

${ }^{6}$ M. Saniga, M. Planat, and H. Rosu, J. Opt. B: Quantum Semiclassical Opt. 6, L19 (2005).

${ }^{7}$ C. A. Fuchs, Quantum Inf. Comput. 4, 467 (2004).

${ }^{8}$ J. Řeháček, B.-G. Englert, and D. Kaszlikowski, Phys. Rev. A 70, 052321 (2004).

${ }^{9}$ W. K. Wootters, Found. Phys. 36, 112 (2006).

${ }^{10}$ I. Bengtsson, e-print arXiv:quant-ph/0406174.

${ }^{11}$ M. Grassl, in Proceedings of the ERATO Conference on Quantum Information Science 2004, Tokyo, Japan, 1-5 September 2004.

${ }^{12}$ J. M. Renes, Quantum Inf. Comput. 5, 80 (2005).

${ }^{13}$ I. Bengtsson and Å. Ericsson, Open Syst. Inf. Dyn. 12, 187 (2005).

${ }^{14}$ R. König and R. Renner, J. Math. Phys. 46, 122108 (2005).

${ }^{15}$ M. Ziman and V. Bužek, Phys. Rev. A 72, 022343 (2005).

${ }^{16}$ D. M. Appleby, J. Math. Phys. 46, 052107 (2005).

${ }^{17}$ A. Klappenecker and M. Rötteler, in Proceedings of 2005 IEEE International Symposium on Information Theory, Adelaide, Australia, 4-9 September 2005, p. 1740.

${ }^{18}$ A. Klappenecker, M. Rötteler, I. Shparlinski, and A. Winterhof, J. Math. Phys. 46, 082104 (2005).

${ }^{19}$ M. Grassl, "Electron. Notes Discrete Math. 20, 151 (2005).

${ }^{20}$ M. A. Ballester, e-print arXiv:quant-ph/0507073?

${ }^{21}$ D. Gross, "Finite phase space methods in quantum information," Diploma thesis, Universität Potsdam, Potsdam, 2005. Available online at http://gross.qipc.org/diplom.pdf.

${ }^{22}$ S. Colin, J. Corbett, T. Durt, and D. Gross, J. Opt. B: Quantum and Semiclassical Opt. 7, S778 (2005).

${ }^{23}$ C. Godsil and A. Roy, Eur. J. Comb. 30, 246 (2009).

${ }^{24}$ S. D. Howard, A. R. Calderbank, and W. Moran, EURASIP J. Appl. Signal Process. 2006, 85685 (2006).

${ }^{25}$ A. J. Scott, J. Phys. A 39, 13507 (2006).

${ }^{26}$ T. Durt, e-print arXiv:quant-ph/0604117.

${ }^{27}$ S. T. Flammia, J. Phys. A 39, 13483 (2006).

${ }^{28}$ M. Grassl, presented at MAGMA 2006 Conference, Technische Universität Berlin, Berlin, Germany, 30 July-2 August 2006. Available at http://magma.maths.usyd.edu.au/Magma/2006.

${ }^{29}$ I. H. Kim, Quantum Inf. Comput. 7, 730 (2007).

${ }^{30}$ D. M. Appleby, Opt. Spect. 103, 416 (2007).

${ }^{31}$ L. Bos and S. Waldron, N. Z. J. Math. 36, 113 (2007).

${ }^{32}$ A. Roy and A. J. Scott, J. Math. Phys. 48, 072110 (2007).

${ }^{33}$ O. Albouy and M. R. Kibler, J. Russ. Laser Res. 28, 429 (2007).

${ }^{34}$ D. M. Appleby, H. B. Dang, and C. A. Fuchs, e-print arXiv:0707.2071.

${ }^{35}$ M. Khatirinejad, J. Algebr. Comb. 28, 333 (2008).

${ }^{36}$ B. G. Bodmann, P. G. Casazza, D. Edidin, and R. Balan, in Proceedings of 42nd Annual Conference on Information Sciences and Systems, CISS 2008, Princeton University, Princeton, NJ, USA, 19-21 March 2008), p. 721.

${ }^{37}$ M. R. Kibler, J. Phys. A 41, 375302 (2008).

${ }^{38}$ I. Bengtsson and H. Granström, Open Syst. Inf. Dyn. 16, 145 (2009).

${ }^{39}$ M. Grassl, presented at Seeking SICs: A Workshop on Quantum Frames and Designs, Perimeter Institute, Waterloo, 2008. Available at http://pirsa.org/08100069.

${ }^{40}$ M. Grassl, Lect. Notes Comput. Sci. 5393, 89 (2008).

${ }^{41}$ M. Fickus, J. Fourier Anal. Appl. 15, 413 (2009).

42 D. M. Appleby, AIP Conf. Proc. 1101, 223 (2009).

${ }^{43}$ C. A. Fuchs and R. Schack, e-print arXiv:0906.2187.

${ }^{44}$ D. M. Appleby, e-print arXiv:0909.5233. 
${ }^{45}$ D. M. Appleby, Å. Ericsson, and C. A. Fuchs, Found. Phys. 41, 564 (2011).

${ }^{46}$ A. J. Scott and M. Grassl, J. Math. Phys. 51, 042203 (2010).

${ }^{47}$ I. Bengtsson and K. Życzkowski,, Geometry of Quantum States (Cambridge University Press, Cambridge, 2006).

${ }^{48}$ C. A. Fuchs, e-print arXiv:quant-ph/0205039.

${ }^{49}$ P. O. Boykin, M. Sitharam, P. H. Tiep, and P. Wocjan, Quantum Inf. Comput. 7, 371 (2007).

${ }^{50}$ N. Mukunda, Arvind, S. Chaturvedi, and R. Simon, Phys. Rev. A 65, 012102 (2001).

${ }^{51}$ G. R. Goodson and R. A. Horn, Linear Algebr. Appl. 430, 1025 (2009).

${ }^{52}$ N. Jacobson, Lie Algebras (Dover Publications, New York, 1979).

${ }^{53}$ J. E. Humphreys, Introduction to Lie Algebras and Representation Theory (Springer-Verlag, New York, 1970).

${ }^{54}$ W. Fulton and J. Harris, Representation Theory (Springer-Verlag, New York, 1991).

55 A. O. Barut and R. Raçzka, Theory of Group Representations and Applications (World Scientific, Singapore, 1986).

${ }^{56}$ W. K. Wootters, Ann. Phys. (NY) 176, 1 (1987).

${ }^{57}$ A. Vourdas, Rep. Prog. Phys. 67, 267 (2004). 